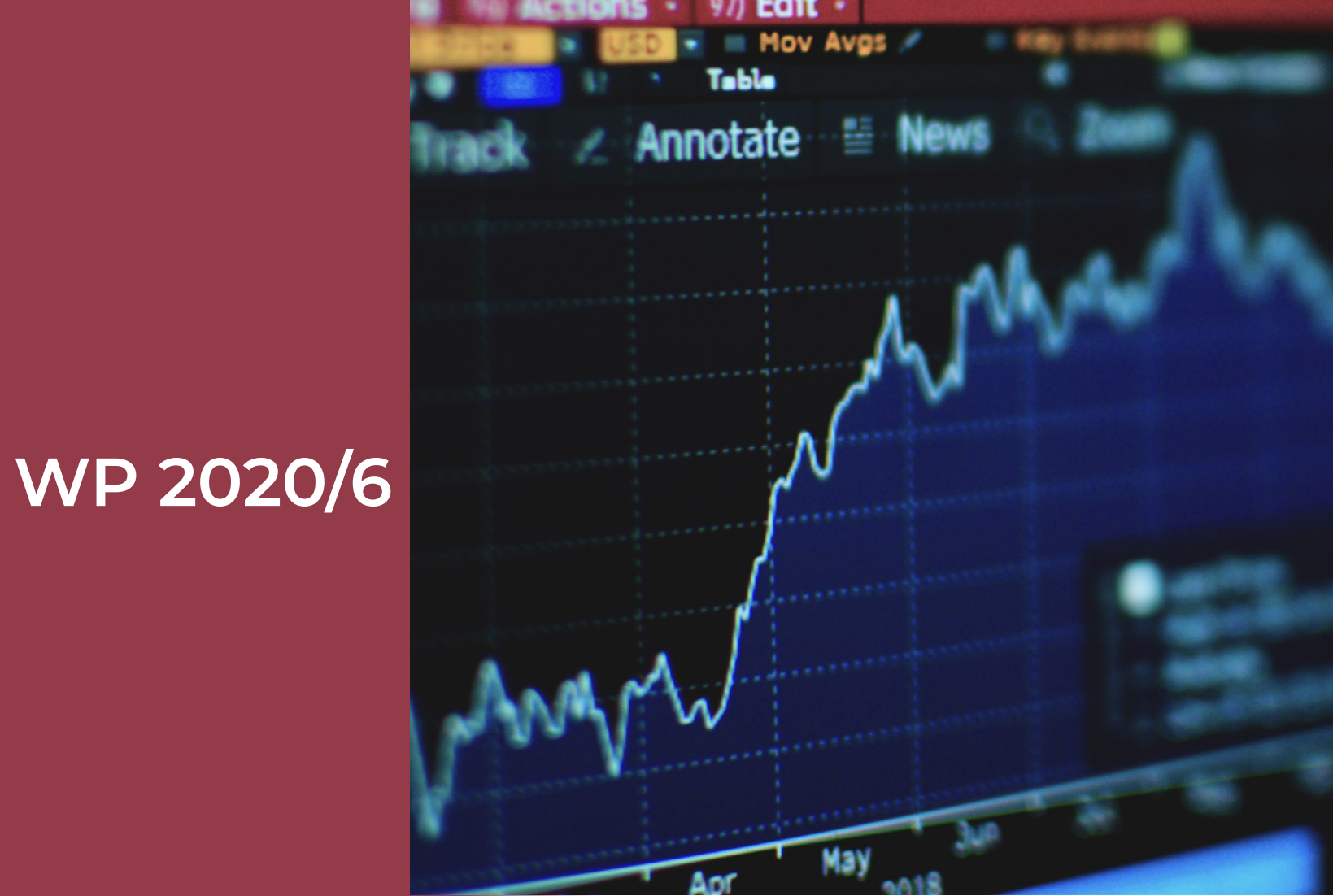

Francesco Roccazzella, Paolo Gambetti and Frédéric Vrins

Optimal and robust combination of forecasts via contrained optimization and shrinkage 


\section{LFIN}

Voie du Roman Pays 34, L1.03.01

B-1348 Louvain-la-Neuve

Tel (32 10) 474304

Email: lidam-library@uclouvain.be

https:/uclouvain.be/en/research-institutes/

lidam/lfin/working-papers.html 


\title{
Optimal and Robust Combination of Forecasts via Constrained Optimization and Shrinkage
}

\author{
Francesco Roccazzella ${ }^{\mathrm{a}, *}$, Paolo Gambetti ${ }^{\mathrm{a}}$, Frédéric Vrins $^{\mathrm{a}}$ \\ ${ }^{a}$ UCLouvain, LIDAM - LFIN, Louvain-la-Neuve, B-1348, Belgium
}

\begin{abstract}
Combining forecasts formed by various models can substantially improve the prediction performances compared to those obtained from the individual models. Standard combination approaches consist in a forecast selection step followed by a weighting scheme. It is not clear, however, which models to include, and how to combine them. This is a central question, having a substantial impact on the quality of the aggregate forecast. We propose a robust method that mitigates estimation uncertainty and implicitly features forecast selection. Our approach relies on constrained optimization with penalty (COP). We take advantage of the equivalence existing between $\mathrm{COP}$ and constrained optimization with shrinkage of the prediction errors' covariance matrix (COS) to determine the optimal L2 penalty, thereby making the economoy of an expensive (and potentially harmful) crossvalidation stage. Our method is tested empirically in a simulation exercise and on two applications in economics. The proposed combination schemes outperform the simple average forecast, trimmed simple average forecast and perform at least as well as the best individual model(s) in the considered cases.

Keywords: Forecasts Combination, Robust Methods, Optimal Combination, Machine Learning
\end{abstract}

\section{Highlights}

- The paper reviews the recent advances in optimization-based forecast combination methods.

- We propose a single-step, fast and robust forecasts combination method with the use of penalized constrained optimization.

- We propose an analytical expression for the optimal shrinkage intensity that improves computational speed and outperforms cross-validation based methods especially when

\footnotetext{
${ }^{*}$ Corresponding author: Voie du Roman Pays 34, B-1348 Louvain-la-Neuve, Belgium.

E-mail addresses: francesco.roccazzella@uclouvain.be, paolo.gambetti@uclouvain.be, frederic.vrins@uclouvain.be
} 
the sample size is limited relative to the number of candidate forecasts.

- Our strategy outperforms the considered benchmarks and the best individual model when predicting housing prices in Boston and the Chicago Fed National Activity Index.

\section{Introduction}

In practice, one can often rely on $m$ different models to get multiple forecasts $\widehat{y}_{1}, \ldots, \widehat{y}_{m}$ of a given random variable $y$. In such a case, two strategies can be considered. A first possibility is to pick up the best prediction, say $\widehat{y}:=\widehat{y}_{i^{\star}}$ where model $i^{\star} \in\{1, \ldots, m\}$ is selected according to some criterion. The alternative is to combine these forecasts into a single prediction, $\widehat{y}:=\Phi\left(\widehat{y}_{1}, \ldots, \widehat{y}_{m}\right)$ using a function $\Phi: \mathbb{R}^{m} \rightarrow \mathbb{R}^{1}$

The first strategy is often disappointing. Indeed, while individual forecasts tend to over-rely on the available data, it is now well-recognized that combining models might be preferable (Atiya, 2020). For example, single models usually over-fit when they are exclusively trained to minimize the in sample loss, while emotions or cognitive biases such as base-rate (Bar-Hillel, 1980) and system neglect (Massey and Wu, 2005) increase the likelihood of over-weighting past information with a negative impact on human predictive ability. This is especially true when it is difficult to identify ex ante the best forecast, when we ignore which among many predictors are relevant, what function relates them to the target variable, or when we are not capable of creating a unified framework that makes optimal use of the aggregate set of information (Clemen, 1989).

Combination methods also make forecasts more robust to noise and miss-specification. For instance, it is known that combining model-based with analysts' forecasts can systematically outperform analysts alone at predicting corporate earnings (Ball and Ghysels, 2018). Also, it is shown in (Lin et al., 2018) that forecasts combinations outperform more traditional models (Fama and French, 1989; Greenwood and Hanson, 2013) in predicting corporate bond returns.

Most combination strategies proceed in two-steps. The first stage consists of removing poorly performing models via trimming or machine learning (ML) methods so as to keep only $k$ models out of $m$ candidates. Then, the selected candidates are combined using a combination scheme, usually in a linear way. This two-step strategy triggers two questions.

First, how to identify the models that should be involved? This amounts to choose a set $\mathcal{I} \subseteq\{1, \ldots, m\}$. Although the selection step can be performed based on rules of thumb (e.g., retaining a fixed percentage of models as in Aiolfi and Favero, 2005), formal tests (e.g.,

\footnotetext{
${ }^{1}$ Throughout the paper we use the terms forecast, prediction and model as synonyms. We note matrices in bold capital letters, while vectors and scalars are respectively bold lowercase and lowercase.
} 
Samuels and Sekkel, 2017) or some measures of interest (Granger and Jeon, 2004, , e.g., information criteria), the choice of the selection criterion makes the decision on whether or not to trim and by how much, more arbitrary. In addition, the risk of including poor candidates or excluding the most advantageous forecast in $\mathcal{I}$ using in sample information in the first step can further deteriorate the results. For instance, forecasts tend to be highly correlated and statistical procedures, such as Lasso, often fail to correctly select the best subset of forecasts (Zhao and Yu, 2006).

Second, how to combine the $k \leq m$ selected models? This amounts to choose a $k$ dimensional combining function $\Phi$. Restricting ourselves to the class of linear combinations $\Phi\left(x_{1}, \ldots, x_{k}\right):=\sum_{i=1}^{k} w_{i} x_{i}$ leads to the aggregated forecast of the form $\widehat{y}=\sum_{i=1}^{k} w_{i} \widehat{y}_{i}$. The equally weighted scheme is popular because of its simplicity; it consists of taking $\boldsymbol{w}=\alpha \mathbf{1}$ where $\mathbf{1}$ is a conformable vector of 1 and $\boldsymbol{w}:=\left(w_{1}, \ldots, w_{k}\right)$ is the $k$-dimensional column vector of weights. Of course, this is a rather naïve method which is expected to be optimal only in very specific cases. ${ }^{2}$ Equal weights are usually valid performers, but they involve an arguably arbitrary choice. Furthermore, including poor performers in an equally weighted combination can substantially worsen the performance of the aggregate forecast (Winkler and Makridakis, 1983).

Alternatively, one can look for more sophisticated schemes. When the individual forecasts are unbiased, the most standard approach consists of minimizing a loss function featuring the aggregated forecast error under the constraint that the weights sum to 1 . In other words, let $\mathbf{y}$ be a $n$-dimensional column vector containing $n$ observations of the variable of interest and $\widehat{\mathbf{Y}}$ be a $n$-by- $m$ matrix of $m$ unbiased candidate forecasts of $\mathbf{y}$. We aim to solve

$$
\boldsymbol{w}^{*}:=\underset{\boldsymbol{w} \in \mathcal{W}}{\operatorname{argmin}}\|\boldsymbol{y}-\widehat{\boldsymbol{Y}} \boldsymbol{w}\|_{2}^{2} \quad \text { where } \quad \mathcal{W}:=\left\{\boldsymbol{w} \in \mathbb{R}^{m} \mid \mathbf{1}^{T} \boldsymbol{w}=1\right\}
$$

Granger and Ramanathan (1984) show that this problem is mathematically equivalent to selecting the weights $\boldsymbol{w}^{*}$ that minimize the variance of prediction error of the aggregate forecast. Its solution is of the well known form $\boldsymbol{w}^{*}=\left[\mathbf{1}^{T} \boldsymbol{\Sigma}^{-1} \mathbf{1}\right]^{-1} \boldsymbol{\Sigma}^{-1} \mathbf{1}$, where $\boldsymbol{\epsilon}:=\boldsymbol{y}-\boldsymbol{Y} \boldsymbol{w}$ and $\boldsymbol{\Sigma}:=\frac{1}{n} \boldsymbol{\epsilon}^{T} \boldsymbol{\epsilon}$ is the positive definite covariance matrix of the prediction errors associated with the selected models. Nevertheless, this approach suffers from a lack of robustness. In particular, it is unstable when $\boldsymbol{\Sigma}$ is poorly estimated; this often occurs due to sample size limitations or considerable background noise (Claeskens et al., 2016).

As a consequence, some approaches have simply ignored how models co-behave and

\footnotetext{
${ }^{2}$ For example, Timmermann (2006) shows that the equally weighted strategy is indeed the optimal combination scheme if and only if individual forecasts have identical variance and identical pair-wise correlation coefficient. In other words, $\forall(i, j \neq i) \in\{1, \ldots, m\}, \sigma_{i}^{2}=\sigma_{j}^{2}=\alpha$ and, $\rho_{i, j}=c$.
} 
focused on estimating combination schemes that rely on candidates' individual losses such as L1, Huber or other losses that help to enhance the robustness to outliers (e.g., the AFTER algorithm of Wei and Yang, 2012).

Diebold and Pauly (1990) instead introduced constrained optimization with penalty (COP) to incorporate prior information into the estimation of the combining weights. As a result, the estimated combining weights are shrunk toward equality but are not forced to be exactly equal. Diebold and Shin (2019) further propose a Lasso-based procedure that estimates the weights imposing some regularization (given that the forecasting record is usually limited relative to the number of candidate forecasts), but that also determines the best subset of forecasts to combine. Nevertheless, despite the one-step conceptualization, the actual implementation of the partially-egalitarian Lasso is in two phases: i) Lasso selects the best set of forecast to combine by penalizing the L1 norm of the weights and ii) estimates the combining weights by penalizing the L1 or L2 norm of the difference between the estimated and equal weights. However, Zhao and Yu (2006) point out that Lasso breaks down if the candidate forecasts are highly correlated, a likely situation when a large number of models is under consideration. The performance of the combined forecast is also sensitive to the choice of the shrinkage intensity both in the first and in the second step. For example, heavy step-2 regularization is eventually required to be competitive with the simple average forecast (Diebold and Shin, 2019). Furthermore, despite they propose subset-averaging procedures that do not require the choice of a tuning parameter, these methods are computationally expensive, raising questions about their practical applicability.

Although more sophisticated than the simple average, the prevailing implementations of COP still suffer from the following weaknesses:

1. they still rely on the a priori identification of the best subset of candidate forecasts;

2. there is no satisfactory rule-of-thumb to determine the penalty strength forcing the user to rely on a time-consuming cross-validation phase;

3. the literature does not provide any justification for the choice of the divergence measure between the estimated weights and the target weighting scheme.

In this paper, we address all these problems by designing a robust single-step framework. Instead of achieving model selection via a pre-processing, we instead consider sparse combination schemes. By doing so, some of the $m$ weights associated with $\Phi$ will vanish, thereby achieving the selection in a natural way. In particular, we restrict the optimal weights to be non-negative, to sum to 1 and, relying on constrained optimization with penalty (COP), we also shrink the solution toward a reference point $\overline{\boldsymbol{w}}$ to further improve robustness (Section 1). Furthermore, building on the correspondence between portfolio strategies that rely on 
shrinkage estimators of the sample covariance matrix (Ledoit and Wolf, 2004a) and the norm-constrained minimum-variance portfolio (DeMiguel et al., 2009), we propose an analytical expression for the "optimal penalty strength" for the COP. This is based on the minimization of the expected value of the Frobenius norm of the difference between the shrinkage estimator of the covariance matrix and the true covariance matrix of prediction errors (Ledoit and Wolf, 2004a).

Finally, in Section 2 and 3, we test the effectiveness of our approach in an extensive simulation study and on two empirical applications. Our comparison features penalized constrained optimization-based methods with various divergence measures and with penalty strength computed either using cross-validation or using the analytical optimal value obtained from the correspondence to the linear shrinkage estimator of the covariance matrix of prediction errors. The last model proves to be a solid challenger: its analytical form makes it much faster than the cross-validation-based alternatives with limited impact on predictive performance. This yields a single-step, fast and robust combination method with explicit penalty strength.

\section{The one-step optimal combination of forecasts}

Before presenting the regularization techniques, let us briefly explain how and why constraining the weights to be non-negative and to sum to 1 implicitly leads to forecasts selection. These constraints have been considered in Jagannathan and Ma (2003), who show that imposing non-negativity and portfolio constraints to the weights also reduces estimation error by inducing a shrinkage-like effect on the sample covariance matrix. Timmermann (2006) recognizes the benefit of this latter aspect for the forecasts combination problem. To the best of our knowledge, these constraints have never been considered for the sake of achieving sparsity, that is, in our context, to perform forecast selection. This is however rather intuitive: if a candidate forecast $j$ has generally a higher covariance with other strategies than other candidates, the optimization procedure will reduce $j$ 's weight accordingly. It will eventually set it to 0 if the marginal contribution of the forecast $j$ to the variance of the aggregate prediction error is always larger than other forecasts' marginal contributions.

\subsection{The optimal combination of forecasts}

We consider the following constrained minimization problem, (the covariance matrix is always assumed to be positive definite in the sequel):

$$
\boldsymbol{w}^{*}:=\underset{\boldsymbol{w} \in \mathcal{W}^{+}}{\operatorname{argmin}} \boldsymbol{w}^{\prime} \boldsymbol{\Sigma} \boldsymbol{w} \quad \text { where } \quad \mathcal{W}^{+}:=\{\boldsymbol{w} \in \mathcal{W} \mid \min \boldsymbol{w} \geq 0\}
$$


This expression is similar to (1) except that the optimization set is $\mathcal{W}^{+}$, the set of nonnegative weights summing to one. The optimal solution is known analytically and can be retrieved by imposing the Karush-Kuhn-Tucker (KKT) conditions (Jagannathan and Ma, 2003):

Proposition 1. The solution to the optimization problem (2) is given by

$$
\boldsymbol{w}^{*}:=\Psi(\boldsymbol{\Sigma})
$$

where

$$
\Psi(\boldsymbol{M}):=\frac{\boldsymbol{M}^{-1} \boldsymbol{\eta}(\boldsymbol{M})}{\boldsymbol{\eta}^{T}(\boldsymbol{M}) \boldsymbol{M}^{-1} \boldsymbol{\eta}(\boldsymbol{M})}
$$

and $\boldsymbol{\eta}(\boldsymbol{M})$ is a conformable column vector whose $j^{\text {th }}$ element is defined as:

$$
\eta_{j}(\boldsymbol{M}):=\left\{\begin{array}{cc}
0 & \text { if }\left[\frac{\boldsymbol{M}^{-1} \mathbf{1}}{\mathbf{1}^{T} \boldsymbol{M}^{-1} \mathbf{1}}\right]_{j} \leq 0 \\
1 & \text { otherwise }
\end{array}\right.
$$

As explained above, the main benefits of (2) are forecasts selection and the reduction of the estimation error. This, however, comes at the cost of misspecification if the constraint $\boldsymbol{w} \in \mathcal{W}^{+}$is violated in population (Green and Hollifield, 1992). In finance, this disadvantage may hurt optimal portfolio allocation, as short and long positions can be perhaps the appropriate solution to the mean-variance portfolio optimization problem. Conversely, in our forecasts combination context, a violation of non-negativity is less relevant: if $w_{j}<0$, the unconstrained solution suggests the existence of a noise forecast, which negatively contributes to the combined predictive performance. Furthermore, we remark that, by imposing non-negativity, the exclusion of a dominated forecast from the combination is operated while computing the weights (one-step selection and combination), which contrasts with the use of trimming methods in a step that precedes the calculation of the weights as in Samuels and Sekkel (2017).

As suggested by Proposition 1, the calculation of the optimal weights requires the estimation of the - inverse of the - covariance matrix of prediction errors (e.g., the sample covariance matrix). If the forecasting record is limited relative to the number of candidates or for large background noise, we cannot exclude the possibility that the solution to (2) is unstable across the sample, thereby impacting the reliability of the optimization procedure.

We solve in Section 1.2 the problem of potential instability of $\boldsymbol{\Sigma}$ by shrinking (2) toward a predetermined weighting scheme or, equivalently, by making use of a robust estimator of the covariance matrix of prediction errors. These approaches are well known in the portfolio optimization literature in finance, but they have not been fully exploited in forecasting. For example, we are not aware of the use of the linear shrinkage estimator of Ledoit and Wolf (2003) in this context, despite Timmermann (2006) acknowledges its potential usefulness 
to improve the estimation of $\boldsymbol{w}^{*}$.

\subsection{Constrained optimization with penalty (COP) on the weighting scheme}

We introduce the COP combination scheme as the solution to an optimization problem where the weights are restricted to both sum to 1 and be non-negative, i.e., $\boldsymbol{w} \in \mathcal{W}^{+}$. Moreover, the minimum-variance objective function is associated with a penalty term, whose purpose is to shrink the solution toward a pre-determined reference scheme $\overline{\boldsymbol{w}}$.

Definition 1 (constrained optimization with penalty - COP). The COP weighting scheme is defined as

$$
\boldsymbol{w}_{\delta}^{*}:=\underset{\boldsymbol{w} \in \mathcal{W}^{+}}{\operatorname{argmin}} \boldsymbol{w}^{T} \boldsymbol{\Sigma} \boldsymbol{w}+\delta\langle\boldsymbol{w}, \overline{\boldsymbol{w}}\rangle,
$$

where the penalty strength $\delta \geq 0$ is the shrinkage intensity and $\langle\cdot, \cdot\rangle:=\mathbb{R}^{m} \times \mathbb{R}^{m} \rightarrow$ $\mathbb{R}^{+},(\boldsymbol{u}, \boldsymbol{v}) \mapsto\langle\boldsymbol{u}, \boldsymbol{v}\rangle$ is a divergence measure between two 1-by-m vectors.

The coefficient $\delta$ is a number that controls the magnitude of the penalty, whereas $\langle\boldsymbol{w}, \overline{\boldsymbol{w}}\rangle$ quantifies the "distance" between $\boldsymbol{w}$ and the reference combination scheme $\overline{\boldsymbol{w}}$. Some possible divergences $\langle\boldsymbol{w}, \overline{\boldsymbol{w}}\rangle$ that will be considered in our simulation study and in the empirical applications are:

- cross-entropy: $\langle\mathbf{u}, \mathbf{v}\rangle=\sum_{j=1}^{m} u_{j} \ln \left(\frac{u_{j}}{v_{j}}\right)$,

- $\mathrm{L} 1:\langle\mathbf{u}, \mathbf{v}\rangle=\|\mathbf{u}-\mathbf{v}\|_{1}$,

- $\mathrm{L} 2:\langle\mathbf{u}, \mathbf{v}\rangle=\|\mathbf{u}-\mathbf{v}\|_{2}^{2}$.

\subsection{The shrinkage direction of combining weights}

Potentially, one can take as $\overline{\boldsymbol{w}}$ in (4) any vector satisfying $\overline{\boldsymbol{w}} \in \mathcal{W}^{+}$; for example, $\overline{\boldsymbol{w}}$ can represent a combination strategy that the forecaster believes to be suitable for a particular predictive problem or can express some prior belief about the exclusion of a model from the set of candidate forecasts. Nevertheless, in practice, we often prefer a fixed reference point to alternatives that require the estimation of individual losses, as the latter can already be subject to estimation error. In particular, given the frequently-found good performance of the simple average both in finite sample (Smith and Wallis, 2009) and in population (Aruoba et al., 2012), equal weights are the natural shrinkage direction for $\boldsymbol{w}$. For this reason, we restrict ourselves to take as reference scheme $\overline{\boldsymbol{w}}$ the simple average, i.e., $\overline{\boldsymbol{w}}_{i}=1 / m$. Note that in this case, the constraint $\left\|\boldsymbol{w}-\frac{1}{m} \mathbf{1}\right\|_{2}^{2}$ collapses to the constraint $\|\boldsymbol{w}\|_{2}^{2}, \forall \boldsymbol{w} \in \mathcal{W}$. This choice also offers some computational advantages since it often simplifies the optimization problem. For example, the COP estimator of the combining weights becomes the following (see Appendix A for a simple proof). 
Proposition 2. In the special case where $\overline{\boldsymbol{w}}=\mathbf{1} / \mathrm{m}$ and $\langle\boldsymbol{u}, \boldsymbol{v}\rangle=\|\boldsymbol{u}-\boldsymbol{v}\|_{2}^{2}$, the COP weighting scheme (4) is given by:

$$
\boldsymbol{w}_{\delta}^{*}=\Psi\left(\boldsymbol{\Sigma}_{\delta}\right) \quad \text { where } \quad \boldsymbol{\Sigma}_{\delta}:=\boldsymbol{\Sigma}+\delta \boldsymbol{I}
$$

\subsection{A closer look at COP combination methods}

In the previous section, we derived a forecasts combination scheme that makes use of constrained optimization with penalty to estimate the optimal set of weights, but also other methods can be adopted. In particular, we can consider shrinkage estimators of $\boldsymbol{\Sigma}$. This approach has been developed in a series of papers by Ledoit and Wolf (2003, 2004a,b, 2012, 2017), who introduced linear and nonlinear shrinkage techniques to reduce the instability of the estimated covariance matrix in a portfolio optimization context. Their idea is to combine the sample covariance matrix (which can be easily computed and it is unbiased in large sample, but prone to estimation error) with a highly structured estimator that contains relatively little estimation error, but tends to be misspecified and biased. Transposing this technique to our forecasts combination problem leads to the following combination scheme.

Definition 2 (Constrained optimization with shrinkage (COS) on the covariance matrix of prediction errors). The COS weighting scheme is defined as

$$
\boldsymbol{w}_{\lambda}^{*}:=\underset{\boldsymbol{w} \in \mathcal{W}^{+}}{\operatorname{argmin}} \boldsymbol{w}^{T} \boldsymbol{\Sigma}_{\lambda} \boldsymbol{w} \quad \text { where } \quad \boldsymbol{\Sigma}_{\lambda}:=(1-\lambda) \boldsymbol{\Sigma}+\lambda \overline{\boldsymbol{\Sigma}}
$$

and $\lambda \in[0,1]$ is the shrinkage intensity and $\bar{\Sigma}$ is the reference covariance matrix.

This problem takes the same form as (2) and its solution is thus simply $\boldsymbol{w}_{\lambda}^{*}=\Psi\left(\boldsymbol{\Sigma}_{\lambda}\right)$.

As explained above, equal weights is a natural direction for shrinkage. Moreover, Timmermann (2006) shows that this is the optimal combining scheme when the covariance matrix of the prediction erroes satisfies $\Sigma_{i i}=\sigma^{2}$ and $\Sigma_{i j, j \neq i}=\rho \sigma^{2}$. This suggests that shrinking towards equal weights could be achieved indirectly, by shrinking the sample covariance matrix toward the one that would be implied if equal weights were indeed the optimal combination strategy. Specifying the target thus simply requires to choose both $\bar{\sigma}^{2}$ (individual prediction error variance) and $\rho$ (pairwise correlation coefficient). Without loss of generality (see Appendix B), we set $\rho=0$ and focus on:

$$
\overline{\boldsymbol{\Sigma}}=\bar{\sigma}^{2} \boldsymbol{I}, \quad \text { where } \quad \bar{\sigma}^{2}=\frac{1}{m} \sum_{i=1}^{m} \sigma_{i}^{2} .
$$

where $\sigma_{i}^{2}$ is the sample estimate of the prediction error variance of model $i$.

Despite following different approaches, the optimal weighting schemes in COP and COS are closely related (2). In particular, in the field of portfolio optimization, DeMiguel et al. 
(2009) show that there is a correspondence between COS and the portfolio that solves the minimum-variance problem subject to the additional constraint that the norm of portfolioweight vector is smaller than a given threshold $\alpha$. We can derive a similar relation also in the context of penalized constrained optimization.

Proposition 3. The COS model with reference covariance matrix $\overline{\boldsymbol{\Sigma}}=\bar{\sigma}^{2} \boldsymbol{I}$ (Def. 2) corresponds to the COP model with reference scheme $\overline{\boldsymbol{w}}=\mathbf{1} / \mathrm{m}$, divergence measure $\langle\boldsymbol{u}, \boldsymbol{v}\rangle=$ $\|\boldsymbol{u}-\boldsymbol{v}\|_{2}^{2}$ and shrinkage intensity $\delta^{*}=\frac{\lambda}{1-\lambda} \bar{\sigma}^{2}$.

The proof of Proposition 3 is trivial. Provided that $\lambda>0^{3}$, one can rescale $\boldsymbol{\Sigma}_{\lambda}$ by $(1-\lambda)$ and obtain $\widetilde{\boldsymbol{\Sigma}}_{\lambda}:=\frac{\boldsymbol{\Sigma}_{\lambda}}{1-\lambda}=\boldsymbol{\Sigma}+\frac{\lambda}{1-\lambda} \bar{\sigma}^{2} \boldsymbol{I}$. Considering $\widetilde{\boldsymbol{\Sigma}}_{\lambda}$ instead of $\boldsymbol{\Sigma}_{\lambda}$ does not impact the COS weighting scheme (2) in the sense that $\boldsymbol{w}_{\lambda}=\Psi\left(\boldsymbol{\Sigma}_{\lambda}\right)=\Psi\left(\widetilde{\boldsymbol{\Sigma}}_{\lambda}\right)$. The equivalence between COP and COS schemes simply results from the fact that, in this specific case, $\widetilde{\boldsymbol{\Sigma}}_{\lambda}$ takes the same form as $\boldsymbol{\Sigma}_{\delta}$ in (5) with $\delta \leftarrow \delta^{*}$. The equivalence holds exactly $\forall \lambda \in[0,1)$. The equivalence also holds in the limiting case since both weighting schemes converge to equal weights, i.e.,

$$
\delta^{*} \stackrel{\lambda \rightarrow 1}{=}+\infty \quad \text { and } \quad \boldsymbol{w}_{\delta} \stackrel{\lambda \rightarrow 1}{=} \frac{1}{m} \mathbf{1}=\boldsymbol{w}_{\lambda}
$$

Proposition 3 has an important implication for the choice of the penalty strength $\delta$. In fact, expressing $\delta=g(\lambda)$ extends the use of an estimated shrinkage intensity in the COS to the COP (with L2 divergence) and vice versa.

\subsection{Estimation of the penalty strength}

The estimate of $\boldsymbol{w}_{\delta}^{*}$ depends on the chosen divergence measure $\langle\cdot, \cdot\rangle$, the reference scheme $\overline{\boldsymbol{w}}$ and the penalty strength, $\delta$. One possibility to select $\delta$ is to rely on standard $k$-fold cross-validation, but more advanced methods such as the forward looking cross-validation technique used in (Diebold and Shin, 2019) could be considered as well. In practice, we estimate the weights for a grid of monotonically increasing $\delta$ and successively test the generalization ability of the solution in a validation set. We eventually choose the penalty strength $\delta$ minimizing the average cross-validation loss. This can mitigate the consequences of a subjective choice of $\langle\cdot, \cdot\rangle$ and $\overline{\boldsymbol{w}}$. Nevertheless, to cut down the computational burden, we also implement early stopping conditions that halt the iterative search of $\delta$ after $s$ steps if the generalization ability of the aggregate forecast does not improve (on average) for a given level of tolerance or when combination weights have converged to the target. Given $\delta$, we solve (4) by imposing the KKT conditions.

However, cross-validation forces us to choose how many observations to retain for validation, which implicitly shrinks the information set for the estimation of the covariance matrix of prediction errors. In fact, when we work with small samples, even few additional

\footnotetext{
${ }^{3}$ The $\lambda=0$ case corresponds to $\delta^{*}=0$, i.e., no shrinkage.
} 
observations can make the difference between singularity and more stable estimates of $\boldsymbol{\Sigma}$. In this regard, Varoquaux (2018) shows that $k$-fold cross-validation can strongly underestimate prediction errors' variance in small samples, inducing forecasters to over-shrink toward equal weights. Therefore, an analytical expression for the shrinkage intensity can improve predictive performance by making a better use of the available data and can also significantly reduce computational cost.

Following Ledoit and Wolf (2004a), we tune the shrinkage intensity $\lambda$ by minimizing the expected value of the Frobenius norm of the difference between the shrinkage estimator of the covariance matrix and the true covariance matrix of prediction errors $\boldsymbol{S}$, i.e.,

$$
\lambda^{*}:=\underset{\lambda \in[0,1]}{\operatorname{argmin}} \mathbb{E}\|(1-\lambda) \boldsymbol{\Sigma}+\lambda \overline{\boldsymbol{\Sigma}}-\boldsymbol{S}\|_{2}^{2} .
$$

By making use of the asymptotic variance (AsyVar) and covariance (AsyCov), the estimator of the optimal solution is (Ledoit and Wolf, 2003):

$$
\begin{gathered}
\widehat{\lambda}^{*}=\frac{\pi-k}{\gamma}, \\
\pi=\sum_{i=1}^{m} \sum_{j=1}^{m} \operatorname{Asy} \operatorname{Var}\left(\sqrt{n} \Sigma_{i, j}\right), \\
k=\sum_{i=1}^{m} \sum_{j=1}^{m} \operatorname{Asy} \operatorname{Cov}\left(\sqrt{n} \Sigma_{i, j}, \sqrt{n} \bar{\Sigma}_{i, j}\right), \\
\gamma=\sum_{i=1}^{m} \sum_{j=1}^{m}\left(\bar{\Sigma}_{i, j}-\Sigma_{i, j}\right)^{2} .
\end{gathered}
$$

Under these assumptions, the estimator of $\lambda^{*}$ becomes:

$$
\widehat{\lambda}^{*}=\frac{\pi-k}{\gamma} \quad \text { where } \pi, k, \gamma \text { are defined as in (10). }
$$

Clearly, the optimal shrinkage intensity $\lambda^{*}$ depends on the target $\bar{\Sigma}$ that we have selected. Therefore, if we impose $\overline{\boldsymbol{\Sigma}}=\bar{\sigma}^{2} \boldsymbol{I}$, a completely inappropriate choice for the target $\bar{\sigma}$ can easily neutralize the COS by coaxing $\lambda^{*}=0$. However, by setting $\bar{\sigma}$ as in (7), we avoid running into this problem since they assume that, a priori, the considered predictive strategies have the same performance (i.e., the same variance of the prediction error) and that possible differences are due to chance. 


\section{Monte Carlo simulations}

We study the behavior of the penalized constrained optimization-based combinations of forecasts in a controlled environment. Specifically, we analyze their sensitivity to various specifications of the variance covariance matrix of prediction errors and to choice of the penalty function $\langle\boldsymbol{w}, \overline{\boldsymbol{w}}\rangle$.

Table 1 summarizes the combination strategies under consideration. The combining weights are restricted to satisfy $\boldsymbol{w} \in \mathcal{W}^{+}$and the shrinkage intensity $\delta$ for COP methods is chosen either via 5 -fold cross-validation (COP1, COP2 and COPE) or by relying on the analytical expression resulting from the COP-COS equivalence (COPS), i.e., taking $\delta \leftarrow \delta^{*}$ where $\lambda \leftarrow \widehat{\lambda}^{*}$ (Proposition 3 and eq. (11)).

Table 1: List of the considered predictive algorithms and forecasts combination methods.

\begin{tabular}{lll}
\hline \hline Combination method & Acronym & Weights \\
\hline Benchmark combination methods & & \\
Simple Average forecast & SA & $\boldsymbol{w}=\mathbf{1} / m$ \\
Trimmed Simple Average forecast & T-SA- $p$ & $\boldsymbol{w}_{i}=1 / \operatorname{card}\left(\mathcal{M}_{p}\right)$ if $i \in \mathcal{M}_{p}, 0$ otherwise. \\
Trimmed-MCS Simple Average forecast & T-MCS- $\alpha$ & $\boldsymbol{w}_{i}=1 / \operatorname{card}\left(\mathcal{M}_{\alpha}\right)$ if $i \in \mathcal{M}_{\alpha}, 0$ otherwise. \\
Loss-based Weighted Average forecast & IL & $\boldsymbol{w}_{i}=\frac{1}{\sum_{j=1}^{m} \frac{1}{\sigma j}}$ \\
Constrained Optimization with Non-Negative Portfolio Weights & CO_NNPW & $\boldsymbol{w}=\Psi(\boldsymbol{\Sigma})$ \\
\hline Constrained optimization with penalty & & \\
COP with L1 penalty & COP1 & $\boldsymbol{w}=\Psi(\boldsymbol{\Sigma})+\delta\|\boldsymbol{w}-\mathbf{1} / m\|_{1}$ \\
COP with L2 penalty & COP2 & $\boldsymbol{w}=\Psi(\boldsymbol{\Sigma})+\delta\|\boldsymbol{w}-\mathbf{1} / m\|_{2}^{2}$ \\
COP with Cross Entropy & COPE & $\boldsymbol{w}=\Psi(\boldsymbol{\Sigma})+\delta \sum_{j=1}^{m} w_{j} \ln \left(\frac{w_{j}}{1 / m}\right)$ \\
COP with L2 penalty and $\delta^{*}$ & COPS & $\boldsymbol{w}=\Psi\left(\boldsymbol{\Sigma}_{\delta^{*}}\right)$ \\
\hline
\end{tabular}

Notes. We denote with $\operatorname{card}(\mathcal{A})$ the cardinality of the set $\mathcal{A}$. For example, $\mathcal{M}_{p}$ the is subset of forecasts containing $m-p$ candidates, such that $\operatorname{card}\left(\mathcal{M}_{p}\right)=m-p$. We denote with T-SA- $p$ the trimmed simple average forecast once the worst $p$ models have been excluded. $\mathcal{M}_{\alpha}$ is the subset of forecasts that are included in the superior set of models with confidence level $\alpha$ (Hansen et al., 2011). We denote with T-MCS- $\alpha$ the simple average forecast derived from the candidate forecasts included in $\mathcal{M}_{\alpha}$. Hence, T-MCS- $\alpha$ represents the simple average forecast that includes only the candidates having at least a probability $\alpha$ of belonging to the superior set of models.

\subsection{Simulation designs}

We model the forecast formed by the $i$-th model as $\widehat{y}_{i}=y+v_{i}$, where $y \sim \mathcal{N}(0,1)$ is the target variable to be predicted and $v_{i}$ stands for the corresponding prediction error. The predictions errors of the $m$ models are assumed to be independent from $y$ and Normally distributed with zero-mean (unbiased forecasts) and covariance matrix $\boldsymbol{\Sigma}_{v}$. More precisely, we generate $t$ predictions from model $i$ as the column vector $\widehat{\boldsymbol{y}}_{i}=\boldsymbol{y}+\boldsymbol{v}_{i}$ where $\boldsymbol{y}$ is a $t$-dimensional column vector with i.i.d. samples of $y$ and $\boldsymbol{v}_{i}$ is the vector of corresponding forecast errors. We define $\widehat{\mathbf{Y}}$ the $t$-by- $m$ matrix whose $i$-th column is set to $\widehat{\boldsymbol{y}}_{i}$. The optimal forecast combination $\widehat{\mathbf{y}}^{*}=\widehat{\mathbf{Y}} \mathbf{c}$ is achieved for some specific weight vector $\mathbf{c}$ which, clearly, 
depends on $\boldsymbol{\Sigma}_{v}$. We consider various forms of $\boldsymbol{\Sigma}_{v}$ for which we know, analytically, the optimal forecasts combination c. Within $\boldsymbol{\Sigma}_{v}$, the $(i, i)$-th element represents the variance of the $i$-th model prediction error, and is restricted to take the form $\alpha_{i}^{2}$. The $(i, j)$-th element takes the form $r \alpha_{i} \alpha_{j}$ where $r$ is a constant pairwise correlation coefficient.

By appropriately tuning $\alpha_{i}$ for $i \in\{1,2, \ldots, m\}$, we focus on various specifications of $\boldsymbol{\Sigma}_{v}$ and control the implied optimal forecasting strategy $\mathbf{c}$ for any given correlation level $r$. Without loss of generality, we consider four correlation levels: $r \in\{0,0.25,0.50,0.90\}$.

Remark. We choose a specific $\boldsymbol{\Sigma}_{v}$ by appropriately tuning $\alpha_{i}$. Let $\widetilde{\boldsymbol{\Sigma}}$ be a square symmetric matrix with ones on the main diagonal and $r$ in the off-diagonal elements. Then, $\forall \boldsymbol{c} \in \mathcal{W}^{+}$and $\forall \widetilde{\boldsymbol{\Sigma}}$, there exists a scaling matrix $\boldsymbol{A}$ such that: $\boldsymbol{c}=\frac{(\boldsymbol{A} \odot \widetilde{\boldsymbol{\Sigma}})^{-1} \mathbf{1}}{\mathbf{1}^{T}(\boldsymbol{A} \odot \widetilde{\boldsymbol{\Sigma}})^{-1} \mathbf{1}}$ and $\boldsymbol{\Sigma}_{v}=\boldsymbol{A} \odot \widetilde{\boldsymbol{\Sigma}}$. The scaling matrix $\boldsymbol{A}$ belongs to the set of positive definite and symmetric matrices of dimension $m \times m$ with diagonal elements $\alpha_{i}^{2}$ and off-diagonal elements $\alpha_{i} \alpha_{j}$. The symbol $\odot$ denotes the Hadamard product. ${ }^{4}$

In this experiment, we compare the considered combination schemes (Table 1) to the optimal forecasting strategy $\boldsymbol{c}$ implied by the true $\boldsymbol{\Sigma}_{v}$. We consider a set of 20 candidate forecasts and four designs of the optimal combination scheme $\boldsymbol{c}$ (see Table 2). In design 1, only the first candidate is included in $\boldsymbol{c}$. In design 2 , only the first two candidates are included in $\boldsymbol{c}$. In design 3, only the first half of the candidates is included in $\boldsymbol{c}$. Design 4 corresponds to the equally-weighted scheme.

Table 2: Optimal combination of forecasts $\boldsymbol{c}$ governing $\boldsymbol{\Sigma}_{v}$ across different designs with $m=20$ candidate models.

\begin{tabular}{cll}
\hline Design & Optimal combination & Description \\
\hline 1 & $\boldsymbol{c}=[1,, 0, \ldots, 0]$ & 1 candidate \\
2 & $\boldsymbol{c}=\left[\frac{1}{2}, \frac{1}{2}, 0, \ldots, 0\right]$ & 2 candidates with equal weights \\
3 & $\boldsymbol{c}=\left[\frac{1}{10}, \ldots, \frac{1}{10}, 0, \ldots, 0\right]$ & 10 candidates with equal weights \\
4 & $\boldsymbol{c}=\left[\frac{1}{20}, \frac{1}{20}, \ldots, \frac{1}{20}\right]$ & 20 candidates with equal weights \\
\hline
\end{tabular}

We simulate 1030 observations (30 training and 1000 testing) for $t=1000$ replications. This is representative of a realistic scenario where only few observations are retained to estimate combining weights and most of the data is used to train the individual candidate forecasts. We refer to the average results in the discussion.

We measure the performance of the combination strategies under consideration (Table 1) with respect to the benchmark $\boldsymbol{c}$. In particular, we compute the relative distance between the root mean square forecast error (RMSFE) of the strategy under consideration and that

\footnotetext{
${ }^{4}$ In the simulation experiment, we set $\alpha_{i}=0.5$ for the candidates that are included and $\alpha_{i}=2$ for the models to be excluded from $\boldsymbol{c}$. We then normalize the specified $\alpha$ s to have a mean of 1 . Some examples of the correspondences between $\boldsymbol{c}$ and $\boldsymbol{\Sigma}_{v}$ are provided in the appendix.
} 
of the optimal strategy, that is, when the combination scheme $\mathbf{w}$ agrees with $\boldsymbol{c}$. Specifically, for the $i^{\text {th }}$ combination scheme we compute the measure $\mu_{i}=\frac{\mathrm{RMSFE}_{i}}{\mathrm{RMSFE}_{\mathbf{c}}}-1$.

\subsection{Simulation results}

Figures 1 includes the results of the four designs. For all considered specifications of $\boldsymbol{\Sigma}_{v}$, we sort the combination schemes (y-axis) on the basis of their average performances across the four correlation levels, which are labeled with the color (yellow $r=0$, orange $r=.25$, red $r=.5$ and black $r=.9$ ). The closer the combination strategy is to the origin of the axes, the closer its average performance is to the optimal forecasting strategy $\boldsymbol{c}$.

In design 1, only one model is included in the optimal forecasting strategy. Hence, combination strategies that operate selection offer a crucial advantage here. However, this holds conditional on selecting the right candidate. On the one hand, we notice that while fixed trimming schemes (T-SAs) perform poorly, T-MCS perform significantly better on average. However, this result clearly depends on the considered correlation level: for example, T-MCS are among the worst predictive strategies when $r=0$ (yellow), but they also rank among the best when $r=0.5$ (red) and $r=0.9$ (black). This was somehow expected: if all models behave similarly, selecting "the right model" or another has limited impact on the RMSFE. On the other hand, optimization-based combinations of forecasts - and in particular COP schemes - always rank among the best combinations both in terms of RMSFE and in terms of variability of their performance under various correlation conditions.

Design 2, including only two candidates in $\boldsymbol{c}$, confirms these findings. In this case, however, T-MCSs perform poorly especially in the no correlation scenario due to inappropriate forecast selection in the training phase. This also explains why T-SA-18 does not rank among the best combination schemes: despite its advantage of discarding exactly the right number of forecasts, it fails to properly identify the right models to combine, which deteriorates predictive power. This is not the case for optimization-based combinations of forecasts: COP1, COP2 and COPE are among the best four combination schemes and COPS ranks first here again.

In design 3, half of the candidates are included in $\boldsymbol{c}$. Therefore, strategies that discard most of the candidate forecasts (e.g., T-SA-18, 16) tend to under-perform compared to more diversified schemes such as SA, IL, T-SA-10, and T-SA-12. The latter two are the best predictive strategies in terms of average RMSFE across correlation schemes, but this result is not unexpected because of their proximity to $\boldsymbol{c}$. In particular, T-SA-10 excludes the exact number of candidates to be discarded in this design (only 10 out of 20 candidates are included in $\boldsymbol{c}$ ): conditional on correctly selecting these models, it is indeed the optimal forecasting strategy a priori. However, this is not always the case. For example, when $r=0$, T-SA-10 fails to correctly identify the models to combine. Contrarily, COPS is 
Design 1

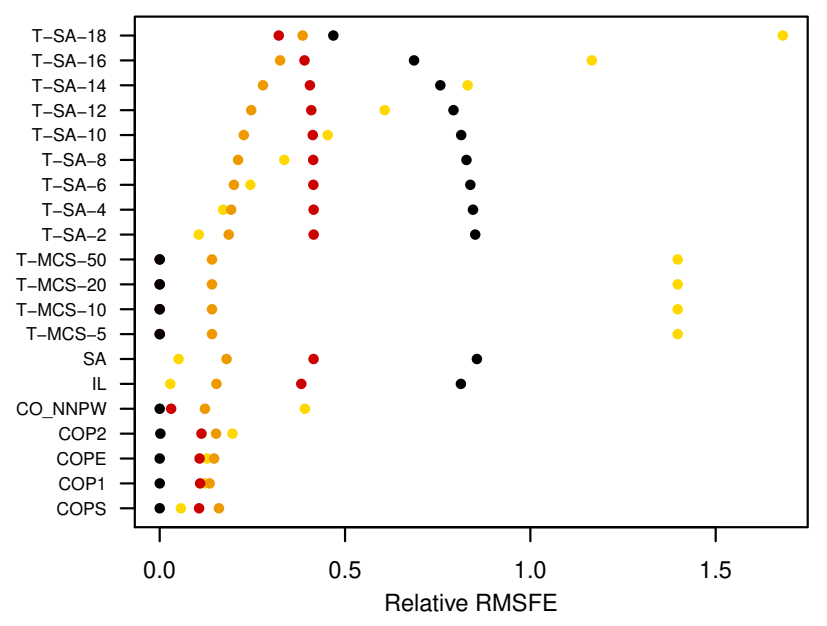

Design 3

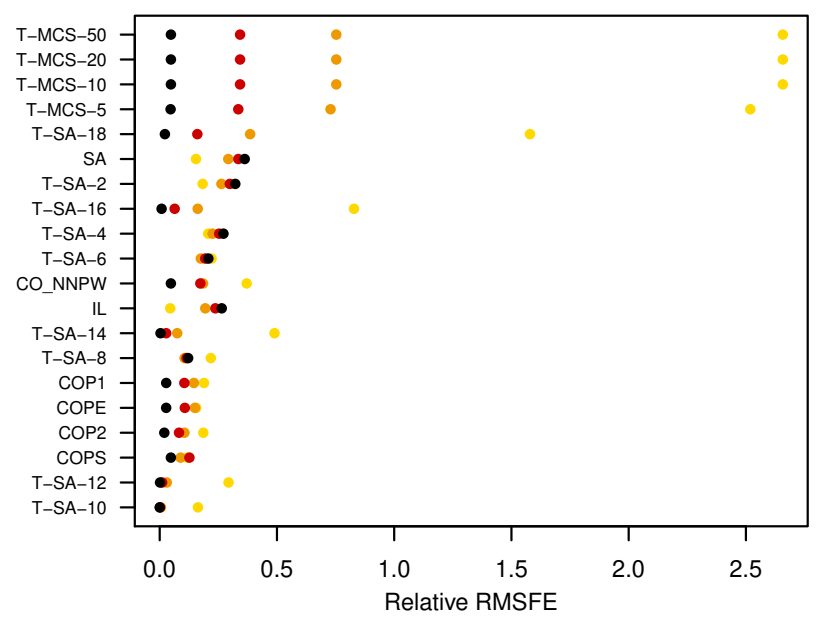

Design 2

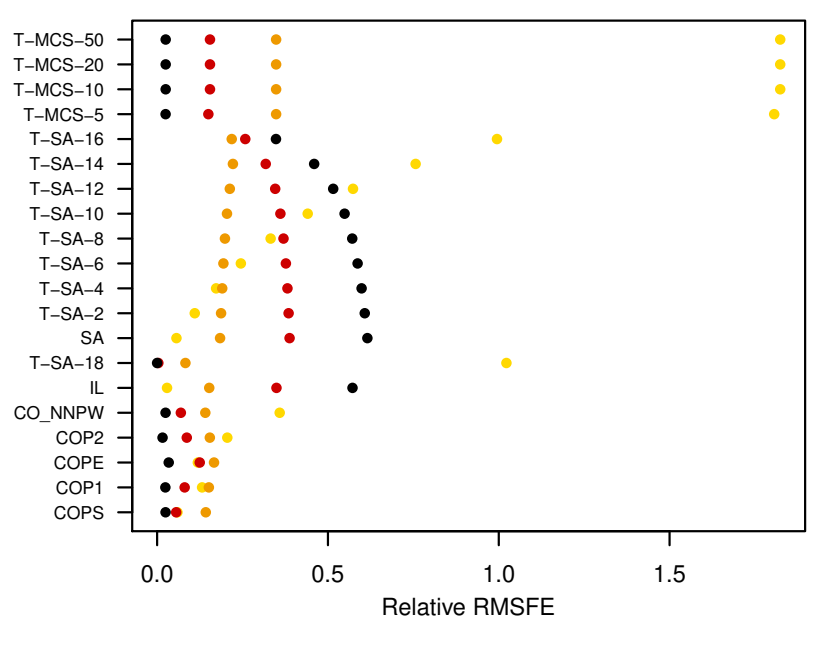

Design 4

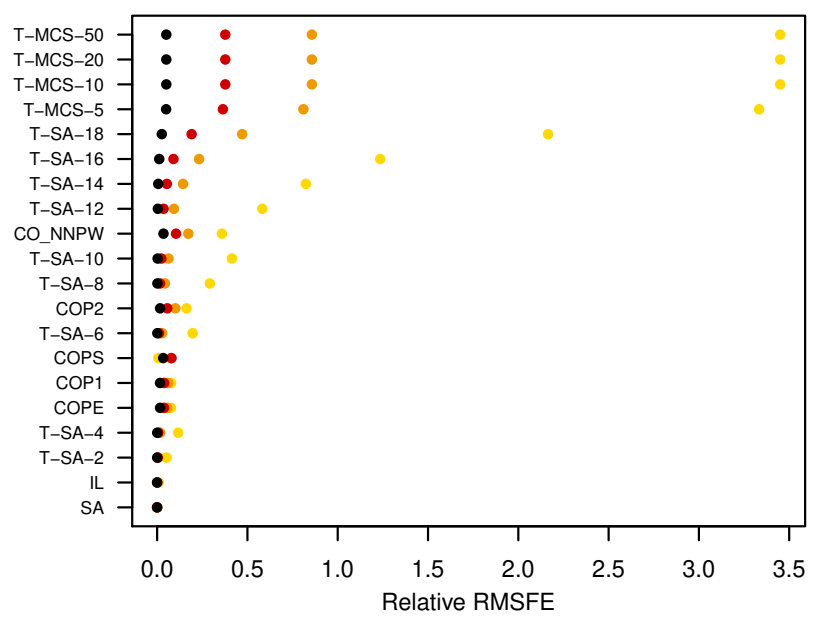

$$
\mathrm{r}=0 \quad \mathrm{r}=.25 \bullet \mathrm{r}=.50 \quad \mathrm{r}=.90
$$

Figure 1: Relative RMSFE with respect to the best forecasting strategy c. Performance of the forecasts combination schemes for various specifications of the forecasts errors' covariance matrix $\boldsymbol{\Sigma}_{\boldsymbol{v}}$, controlled via the designs described in Table 2. Combination schemes are ordered according to their average performances across correlation levels. The relative RMSE is computed with respect to the one of the true optimal forecasts combination.

the forecasting strategy that minimizes the variability of RMSFE across all considered correlation levels.

Eventually, we confirm the previous findings also with respect to design 4, where all models are included with equal weights in $\boldsymbol{c}$. On the one hand, IL and (obviously) SA converge to $\boldsymbol{c}$, with T-SA-2,4 closely following. However, T-SA-2,4 exclude respectively two 
or four models from the combination despite they should be all included in $\boldsymbol{c}$. This loss of information explains the poor performances of T-SA-2,4 when $r=0$, i.e., when candidate forecasts behave differently. On the other hand, COPE, COP1 and COPS perform remarkably well and still very close to the optimal forecasting strategy $\boldsymbol{c}$. COP2's performance is still comparable, but worse than the other COP schemes. Nonetheless, COP2 still significantly outperforms CO_NNPW, aggressive trimming schemes such as T-SA-16,18 and the trimming rules relying on the model confidence set.

This simulation study sheds light over five aspects of great practical relevance for the use of combination strategies in forecasting. First, all optimization-based strategies perform remarkably well, especially compared to rule-based combination strategies for any level of correlation and in all considered designs.

Second, it is indeed difficult to identify a unique rule-based combination method that is optimal under various specifications of the covariance matrix of prediction error. In particular, inappropriately selecting the candidate forecasts to combine has an important negative impact on predictive performance.

Third, optimization-based schemes that rely on non-negative weights that sum to one are solid challengers to rule-based combinations, especially because of their flexibility. In particular, COP methods always rank among the best combination strategies and limit the impact of subjective choices on the number of models to discard. A subjective choice of the penalty function (L1, L2 and cross entropy) does not sensibly affect predictive performances.

Fourth, if we define model risk as the expected variability in performance due to a variation of the correlation conditions and underlying covariance structure of prediction errors, it becomes clear that COPS yields the minimum model risk relative to the considered combination strategies.

Fifth, also compared to COP1, COP2 and COPE, COPS' analytical expression for the shrinkage intensity offers two advantages. Firstly, COPS is significantly faster than other COP methods, because it does not require a computationally expensive cross-validation

procedure. Secondly and most importantly, COPS performs at least as well as the best empirically validated COP method. In fact, because of its analytical expression for the shrinkage intensity, COPS does not need to reduce the sample size (for creating a validation fold for the shrinkage intensity), but can make full use of the training set to estimate the covariance of prediction errors. This provides a crucial advantage for estimating more precisely the combining weights.

\section{Empirical study}

In the empirical study, we consider two prediction problems taken from economics. In these applications, we train the individual algorithms using $70 \%$ of the data, build forecasts combinations on $15 \%$ and keep the remaining $15 \%$ for testing. Results are robust to different 
sizes of the estimation windows. In the following sections, we present the evaluation criteria we use for comparing the considered predictive strategies and the predictive algorithms that we use to form the candidate forecasts.

\subsection{Evaluation criteria}

We must define some evaluation criteria to perform the comparison among predictive strategies. We consider the RMSFE and two statistical procedures for this purpose, namely the Diebold-Mariano test (hereafter DM, Diebold and Mariano, 1995) and the Model Confidence Set (hereafter MCS, Hansen et al., 2011).

The DM tests whether one model is superior to another for a given confidence level. Formally, let the loss difference between forecast $i$ and $j$ for the observation $t$ be $d_{i, j}^{t}=\left(e_{i}^{t}\right)^{2}-$ $\left(e_{j}^{t}\right)^{2}$. The DM tests whether $H_{0}: D M=\frac{\mathbb{E}\left[d_{i, j}\right]}{\sigma\left[d_{i, j}\right]}=0$, with $D M \sim \operatorname{standard}$ t-student $(d f)$.

The MCS tests whether a subset of methods enters jointly in the superior set of models by repeatedly testing the null hypothesis of equal predictive performance with significance level $\alpha$. Let $\mathcal{M}_{0}$ be the set of all forecasting models (both individual candidates and forecasts combinations) and $\mathcal{M}^{*}$ be the superior set of models. Formally, the MCS tests $H_{0}: \mathbb{E}\left[d_{i, j}\right]=0, \forall i, j$. If the null hypothesis is rejected, the procedure eliminates the model with the greatest relative loss from the set $\mathcal{M}$. This procedure is sequentially repeated until the null hypothesis is not rejected at the chosen probability level $\alpha$. We compute the MCS p-values via bootstrap (3000 replications) and using The Oxford MFE Toolbox publicly available at https://www.kevinsheppard.com/code/matlab/mfe-toolbox/.

\subsection{Individual predictive algorithms}

We consider individual forecasts from 13 standard predictive algorithms, which are publicly available on software such as MatLab. Table 3 lists the predictive algorithms used to form candidate forecasts.

For all the considered models, we tune the respective hyper-parameters via 10-fold cross-validation. Ensembles methods such as gradient boosted regression trees (GBRT), random forests $(\mathrm{RF})$ and neural networks $(\mathrm{NN})$ consist respectively of 200, 400 and 5 base learners. This choice is an appealing compromise between computational time and out of sample results. Furthermore, data sets are standardized (unitary variance and zero-mean) and predictors are cross-sectionally normalized to be within $[-1,1]$. These procedures are standard in the field of machine learning; see for instance $\mathrm{Gu}$ et al. (forthcoming) for a financial case. Combination methods that rely on validation to estimate the shrinkage intensity, use 5 -folds cross-validation.

\subsection{Empirical study I: Boston housing prices}

The first application consists in predicting Boston housing prices using 14 attributes (e.g., per capita crime rate, average number of rooms per dwelling or $\%$ lower status of 
Table 3: List of the considered predictive algorithms.

\begin{tabular}{lll}
\hline \hline Predictive model & Abbreviation & MatLab function \\
\hline Generalized linear models & & \\
Ordinary least squares & OLS & \\
Lasso & L1 & lasso \\
Ridge & L2 & lasso \\
Elastic net & EN & lasso \\
Linear SVM with L1 regularization & svmL1 & fitrlinear \\
Linear SVM with L2 regularization & svmL2 & fitrlinear \\
Partial least squares & PLS & plsregress \\
\hline Regression trees & & \\
Gradient boosted trees & GBRT & fitrensemble \\
Random forests & RF & fitrensemble \\
\hline Feedforward neural networks (\# of neurons per layer) & & \\
1 Hidden layer $(8)$ & NN1 & fitnet \\
1 Hidden layer $(16)$ & NN2 & fitnet \\
2 Hidden layers $(8-4)$ & NN3 & fitnet \\
2 Hidden layers $(16-8)$ & NN4 & fitnet \\
\hline
\end{tabular}

the population). This data set consists of 507 observations and it is freely available at http://lib.stat.cmu.edu/datasets/boston. Despite it was originally studied to provide quantitative estimates of the willingness to pay for air quality (Harrison and Rubinfeld, 1978), nowadays it is commonly used in the literature to benchmark predictive algorithms.

Table 4 provides a comparison of the performances, DM and MCS statistics, RMSFE, computational time (Time) and the Concentration Index (CI). The first section of each table gives the results of the DM test for all models (rows) with respect to the forecasts combination schemes (headers). The symbol $<, \ll$ or $\lll$ located to row $i$ and column $j$ indicates that model $j$ is better than model $i$ with confidence $90 \%, 95 \%$ and $99 \%$, respectively. The symbol "=" means that the difference in performance is not significant at $10 \%$. Time indicates the computational cost of optimization-based combination strategies measured in seconds, while the CI measures the average degree of diversification of the strategy. We also indicate the algorithms providing the best predictive performance (lowest RMSFE) while combing forecasts and in the testing phase with + and $*$, respectively.

Looking at the RMSFEs, we notice that despite using standard and publicly available algorithms, our results are competitive with other predictive exercises on the same data set (average RMSFE $\approx .36$, see https://www . kaggle.com). However, the benefit of combining forecasts, and in particular optimization-based methods, is what clearly stands out. Indeed, COPE and COPS yield the lowest RMSFEs, also outperforming NN4 and all the considered trimmed schemes (both T-SAs and T-MCSs). However, this difference in predictive performances between COP is not statistically significant. We can easily understand why this happens by looking at the Concentration Index (CI) and compare COP and T-MCSs. We 
Table 4: Predicting Boston housing prices, out of sample predictive performance.

\begin{tabular}{|c|c|c|c|c|c|c|c|c|}
\hline & \multicolumn{4}{|c|}{ Diebold-Mariano } & \multirow{2}{*}{$\begin{array}{c}\text { MCS } \\
i \in \mathcal{M}^{*}\end{array}$} & \multirow[t]{2}{*}{ RMSFE } & \multirow{2}{*}{$\begin{array}{l}\text { Time } \\
\text { (sec) }\end{array}$} & \multirow[t]{2}{*}{$\mathrm{CI}$} \\
\hline & $\mathrm{j}: \mathrm{COP} 1$ & COP2 & COPE & COPS & & & & \\
\hline $\mathrm{NN} 4+^{*}$ & $=$ & $=$ & $=$ & $=$ & * & .3312 & & \\
\hline$-\overline{\mathrm{SA}}$ & ஊ & ఋ & ఋ & ஊ & & $\overline{4} \overline{6} 1 \overline{1}$ & & \\
\hline$\overline{\mathrm{T}}-\overline{\mathrm{SA}}-\overline{3}$ & ఋ & $\lll$ & $\lll$ & ఋ & & $\overline{4} \overline{3} 1 \overline{4}$ & & $\overline{15.8 \%}$ \\
\hline T-SA-4 & $\lll$ & $\lll$ & $\lll$ & $\lll$ & & .4112 & & $19.2 \%$ \\
\hline T-SA-5 & $\lll$ & $\lll$ & $\lll$ & $\lll$ & & .3878 & & $22.8 \%$ \\
\hline T-SA-6 & $\lll$ & $\lll$ & $\lll$ & $\lll$ & & .3644 & & $26.8 \%$ \\
\hline T-SA-7 & $=$ & $\ll$ & $\lll$ & $\lll$ & & .3405 & & $31.2 \%$ \\
\hline T-SA-8 & $=$ & $=$ & $=$ & $=$ & $* * *$ & .3259 & & $36.5 \%$ \\
\hline T-SA-9 & $=$ & $=$ & $=$ & $=$ & & .3369 & & $43.3 \%$ \\
\hline T-SA-10 & $=$ & $=$ & $=$ & $=$ & $*$ & .3357 & & $52.7 \%$ \\
\hline T-SA-11 & $=$ & $=$ & $=$ & $=$ & $*$ & .3285 & & $67.7 \%$ \\
\hline$\overline{\mathrm{T}}-\overline{\mathrm{M}} \overline{\mathrm{CS}}-\overline{20 \%}$ & $=$ & $=$ & $=$ & $=$ & $\bar{*}$ &.$\overline{3} \overline{3} \overline{7}$ & & $\overline{5} 2 . \overline{7} \%$ \\
\hline T-MCS- $40 \%$ & $=$ & $=$ & $=$ & $=$ & $*$ & .3285 & & $67.7 \%$ \\
\hline T-MCS-60\% & $=$ & $=$ & $=$ & $=$ & $*$ & .3312 & & $100 \%$ \\
\hline T-MCS- $80 \%$ & $=$ & $=$ & $=$ & $=$ & * & .3312 & & $100 \%$ \\
\hline$----\overline{\mathrm{I}} \mathrm{L}^{-}$ & $\lll$ & $\lll$ & $\lll$ & « & &.$\overline{4} 29 \overline{2}$ & & $5.7 \overline{\%}$ \\
\hline${ }^{-} \overline{\mathrm{C}} \mathrm{O} \_\overline{\mathrm{N}} \overline{\mathrm{N}} \overline{\mathrm{P}} \overline{\mathrm{W}}$ & $=$ & « & « & « & $\bar{*}$ &.$\overline{3} \overline{4} \overline{0}$ &.$\overline{0} \overline{80} \overline{1}^{-}$ & $\overline{4} 1 . \overline{9} \%$ \\
\hline COP1 & & $\lll$ & $\lll$ & $\lll$ & $*$ & .3392 & 53.1800 & $32.1 \%$ \\
\hline COP2 & & & $\lll$ & $=$ & $*$ & .3307 & 31.3582 & $32.7 \%$ \\
\hline COPE & & & & $=$ & $* * *$ & .3223 & 52.8043 & $40.5 \%$ \\
\hline COPS & & & & & $* * *$ & .3253 & .0504 & $42.6 \%$ \\
\hline
\end{tabular}

Notes. We denote with $*$ the best individual model in testing phase, while we indicate with + the best model in the combination phase. Symbols $<, \ll$ and $\lll$ indicate that model $j$ (column index) surpasses model $i$ (row index) at a confidence level of $90 \%, 95 \%$ and $99 \%$, respectively. Failure to reject the null hypothesis of equal predictive performance at $10 \%$ significance level is indicated with "=". The MCS considers significance level of $1 \%, 5 \%$ and $10 \%$ (respectively $* * *$ and $* * *$ ), providing the minimum probability that model $i$ is included in the superior set of models. The Concentration Index is defined as $\mathrm{CI}=k^{-1}\|\boldsymbol{w}-\mathbf{1} / m\|_{2}$, where $k=\left\|[1,0, \ldots, 0]^{T}-\mathbf{1} / m\right\|_{2}$.

notice that T-MCS-60\% and T-MCS- $80 \%$ actually operate forecast selection and identify NN4 as the best forecast. This is not the case for COP strategies: these latter provide better or comparable predictive performances, but are also more diversified. This significantly reduces model risk. With respect to T-SAs, the difference in predictive performance strongly depends on how many candidate forecasts we discard in the trimming phase. In this sense, we confirm the results of Aiolfi and Favero (2005) and Granger and Jeon (2004): among the class of trimmed simple average strategies, those discarding most of the candidate forecasts perform best. Nevertheless, we cannot draw a clear pattern about the optimal number of models to discard in the trimming phase. For example, while T-SA- 8 is among the best performers and it is included with $10 \%$ in the superior set of models, T-SA-7 and T-SA-9 have less than $1 \%$ probability of being included. Overall, the choice of the number of can- 
didate forecasts to consider in T-SA is purely subjective. On the contrary, COP methods do not require any preliminary decision about whether to trim and by how much, but they only rely on the estimated covariance matrix of prediction errors to operate selection and combination in one shot.

Table 5: Predicting Boston housing prices, combination weights.

\begin{tabular}{lccccccccccccc}
\hline \hline & OLS & L1 & L2 & SvmL1 & SvmL2 & EN & PLS & RF & GBRT & NN1 & NN2 & NN3 & NN4 \\
\hline SA & .077 & .077 & .077 & .077 & .077 & .077 & .077 & .077 & .077 & .077 & .077 & .077 & .077 \\
T-SA-3 & .100 & .100 & .100 & .100 &. &. & .100 &. & .100 & .100 & .100 & .100 & .100 \\
T-SA-4 & .111 & .111 & .111 &. &. &. & .111 &. & .111 & .111 & .111 & .111 & .111 \\
T-SA-5 & .125 & .125 & .125 &. &. &. &. &. & .125 & .125 & .125 & .125 & .125 \\
T-SA-6 & .143 &. & .143 &. &. &. &. &. & .143 & .143 & .143 & .143 & .143 \\
T-SA-7 &. &. & .167 &. &. &. &. &. & .167 & .167 & .167 & .167 & .167 \\
T-SA-8 &. &. &. &. &. &. &. &. & .200 & .200 & .200 & .200 & .200 \\
T-SA-9 &. &. &. &. &. &. &. &. &. & .250 & .250 & .250 & .250 \\
T-SA-10 &. &. &. &. &. &. &. &. &. & .333 & .333 &. & .333 \\
T-SA-11 &. &. &. &. &. &. &. &. &. & .500 &. &. & .500 \\
T-MCS-20\% &. &. &. &. &. &. &. &. &. & .333 & .333 &. & .333 \\
T-MCS-40\% &. &. &. &. &. &. &. &. &. & .500 &. &. & .500 \\
T-MCS-60\% &. &. &. &. &. &. &. &. &. &. &. &. & 1 \\
T-MCS-80\% &. &. &. &. &. &. &. &. &. &. &. &. & 1 \\
IL & .068 & .068 & .068 & .066 & .065 & .063 & .067 & .060 & .082 & .101 & .098 & .092 & .102 \\
CO_NNPW &. &. &. &. &. &. & .112 & .042 & .340 & .294 &. & .107 & .106 \\
COP1 & .062 &. &. &. &. &. & .063 & .063 & .313 & .125 & .125 & .125 & .125 \\
COP2 & .017 & .018 &. &. &. &. & .065 & .067 & .277 & .148 & .063 & .125 & .219 \\
COPE & .014 & .014 &. &. &. &. & .043 & .054 & .296 & .106 & .085 & .049 & .338 \\
COPS &. &. &. &. &. &. & .095 & .030 & .250 & .125 & .125 &. & .375 \\
\hline
\end{tabular}

Table 5 reports the combining weights and confirms that constraining weights to be non-negative operates forecast selection. However, the use of different divergence measures for COP implies different optimal combination schemes. The reason of this relates to the nature of the forecasts combination problem. In fact, individual models are trained on the same set of information to minimize the mean squared error and, with the exception of non-parametric techniques, they rely on similar modeling assumptions (e.g., linearity for OLS, L1, L2, EN and PLS). Therefore, they also produce highly correlated forecasts, which results in nearly singular estimated covariance matrices of prediction errors. This problem has important consequences on the estimation of the optimal solution: similarly to multicollinearity in a linear regression model, the optimization algorithm has difficulties to identify a unique set of weights that minimizes the variance of the aggregate forecast error. However, despite single weights can differ across the plethora of COP methods as well as CO_NNPW, the combining strategies largely agree in their selection feature. While svmL1, svmL2,EN and L2 are always discarded, PLS, NNs, GBRT and RF are generally 
included in the combination and they are the main contributors to the aggregate forecast.

\subsection{Empirical study II: Chicago Fed National Activity Index}

The second application aims to predict the Chicago Fed National Activity Index (CFNAI), a monthly index measuring the current economic activity for United States. The index, publicly available at https://fred.stlouisfed.org/series/CFNAI, is a weighted average of 85 monthly indicators of national economic activity (e.g., production and income, employment, personal consumption, housing, sales and inventories). The CFNAI is constructed to have an average value of zero and a standard deviation of one. Zero values indicate that the US economy is expanding at its historical trend rate of growth; negative values indicate that the economy is below-average growth; positive values indicate above-average growth.

We predict the current level of CFNAI using a large data set of backward and forward looking variables. As for the backward looking category, we retrieve variables from the Federal Reserve Bank of St. Louis' monthly data set (FRED-MD) and transform the original series according to the work of McCracken and Ng (2016). We also include measures of macroeconomic, financial and real uncertainty from Jurado et al. (2015) and Ludvigson et al. (2019). We instead retrieve forward looking variable from the Blue Chips Financial forecasts (BCFFs) database. This database relies on a survey of professional forecasters that includes the expected term structure of U.S. interest rates, expected real GDP, price level (GDP price deflator and Consumer Price Index) and the expected level of the Major Currency Index. BCFFs also include the average expected yield for home mortgages, and the yield of Aaa and Bbb-rated corporate bonds. We take the first three principal components to summarize the information of the term structure of expected yields. We further compute the standard deviation and the conditional asymmetry (Bowley, 1920) to capture, respectively, the dispersion and the skewness of the empirical distribution of forecasts. We count 125 predictive variables. Our sample starts in January 2001 and stops in May 2018 (209 monthly observations). Using the same notation of the previous sections, Table 6 presents RMSFE, DM, MCS, the Concentration Index and computational time measured in seconds.

The results of this application confirm and strengthen our previous findings. EN is the individual model that would have been selected if we were looking at the RMSFE in the combining phase. However, in the testing set, the ranking of the candidate forecasts changes. EN does not provide the lowest RMSFE error anymore and is significantly outperformed by L2. For this reason, in contrast with predicting Boston housing prices, T-MCS- $40 \%,-60 \%,-80 \%$ fail to select the best ex post candidate forecast. This has an important impact on their predictive performance. On the contrary, COP strategies are still 
Table 6: Predicting the Chicago Fed National Activity Index (CFNAI), out of sample predictive performance.

\begin{tabular}{|c|c|c|c|c|c|c|c|c|}
\hline & \multicolumn{4}{|c|}{ Diebold-Mariano } & \multirow{2}{*}{$\begin{array}{c}\text { MCS } \\
i \in \mathcal{M}^{*}\end{array}$} & \multirow[t]{2}{*}{ RMSFE } & \multirow{2}{*}{$\begin{array}{l}\text { Time } \\
\text { (sec) }\end{array}$} & \multirow[t]{2}{*}{ CI } \\
\hline & COP1 & $\mathrm{COP} 2$ & $\mathrm{COPE}$ & COPS & & & & \\
\hline$\overline{\mathrm{L} 2}{ }^{*}$ & $=$ & $\overline{=}$ & $=$ & $=$ & $* * *$ & .3534 & & \\
\hline EN+ & $\lll$ & $\lll$ & $\lll$ & $\lll$ & & .4291 & & \\
\hline$-\overline{\mathrm{SA}^{-}}$ & $\overline{=}$ & $\lll$ & $\lll$ & $\ll$ & &.$\overline{3} 6 \overline{8} 0$ & & \\
\hline$\overline{\mathrm{T}}-\overline{\mathrm{SA}}-\overline{3}$ & $=$ & $-\ddot{=}$ & $=$ & $=$ & $* \bar{*}$ &.$\overline{3} 4 \overline{7} \overline{2}$ & & $\overline{15.8 \%}$ \\
\hline T-SA-4 & $=$ & $\lll$ & $=$ & $\ll$ & & .3736 & & $19.2 \%$ \\
\hline T-SA-5 & $=$ & $<$ & $=$ & $<$ & $*$ & .3617 & & $22.8 \%$ \\
\hline T-SA-6 & $=$ & $=$ & $=$ & $<$ & & .3603 & & $26.8 \%$ \\
\hline T-SA-7 & $=$ & $<$ & $=$ & $<$ & & .3652 & & $31.2 \%$ \\
\hline T-SA-8 & $=$ & $\ll$ & $=$ & $\ll$ & & .3836 & & $36.5 \%$ \\
\hline T-SA-9 & $\lll$ & $\lll$ & $\lll$ & $\lll$ & & .4235 & & $43.3 \%$ \\
\hline T-SA-10 & $\ll$ & $\lll$ & $\lll$ & $\lll$ & & .4040 & & $52.7 \%$ \\
\hline T-SA-11 & $\ll$ & $\lll$ & $\lll$ & $\lll$ & & .4070 & & $67.7 \%$ \\
\hline$\overline{\mathrm{T}}-\overline{\mathrm{M}} \overline{\mathrm{C}} \overline{\mathrm{S}}-\overline{2} \mathrm{0} \overline{\%}$ & $\bar{\ll}$ & $\bar{\lll}$ & ஊ & $\bar{\lll}$ & &.$\overline{4} 0 \overline{4} \overline{0}$ & & $\overline{52.7 \overline{\%}}$ \\
\hline T-MCS- $40 \%$ & $\lll$ & $\lll$ & $\lll$ & $\lll$ & & .4291 & & $100 \%$ \\
\hline T-MCS-60\% & $\lll$ & $\lll$ & $\lll$ & $\lll$ & & .4291 & & $100 \%$ \\
\hline T-MCS- $80 \%$ & $\lll$ & $\lll$ & $\lll$ & $\lll$ & & .4291 & & $100 \%$ \\
\hline$----\overline{\mathrm{I}} \mathrm{L}$ & $\overline{=}$ & $-\overline{-}$ & $=$ & $<$ & &.$\overline{3} 6 \overline{4}$ & & $\overline{6} . \overline{9} \overline{\%}^{-}$ \\
\hline${ }^{-} \overline{\mathrm{C}} \overline{\mathrm{O}}-\overline{\mathrm{N}} \overline{\mathrm{N}} \overline{\mathrm{P}} \overline{\mathrm{W}}$ & $\gg$ & $=$ & $=$ & $=$ & $\bar{*} *^{-}{ }^{-}$ &.$\overline{3} 2 \overline{9} \overline{5}$ & $\overline{1} \overline{2} . \overline{8} 0 \overline{1} \overline{1}$ & $\overline{43} . \overline{7} \overline{\%}$ \\
\hline COP1 & & $\lll$ & $\lll$ & $\ll$ & & .3680 & 110.2757 & $0.1 \%$ \\
\hline COP2 & & & $\ggg$ & $=$ & $* * *$ & .3360 & 144.5386 & $11.0 \%$ \\
\hline COPE & & & & $<$ & & .3566 & 207.4108 & $2.84 \%$ \\
\hline COPS & & & & & $* * *$ & .3296 & .0352 & $36.0 \%$ \\
\hline
\end{tabular}

Notes. We refer to Table 4 for the details on the notation.

particularly diversified: this mitigates model risk and, in particular, the cost of an error when operating forecast selection.

CO_NNPW, COP2 and COPS provide the lowest RMSFE, outperforming the ex post and ex ante best candidate forecasts (L2* and EN+ respectively). CO_NNPW, COP2, COPS also belong to the superior set of model with at least $10 \%$ confidence, while COP1 and COPE are not included at any confidence level. In this case, the choice of the penalty function matters for predictive performance. Nevertheless, looking at the DM statistics, COPE and COP1 perform at least as well as the best L2, T-SAs, T-MCSs and significantly outperform the best ex ante individual model EN. Among COP schemes, differences in computational cost are also evident: the estimation of the shrinkage intensity by 5 -fold cross-validation has a relevant impact on Time. In fact, despite the implementation of early stopping conditions, COP1, COP2 and COPE require 15 times the computational cost of CO_NNPW. On the contrary, COPS is considerably faster.

Eventually, Table 7 gives an overview on the estimated weights. We find additional 
evidence that COPS is also sparse, i.e., it only combines a subset of the available candidate forecasts. Nevertheless, COPS remains also more diversified than CO_NNPW, which excludes an additional forecast, and than most of the trimmed simple average schemes. On the contrary, COP1, COP2 and COPE do not operate forecast selection in this application: all models are included in the optimal weighting scheme. In particular, COP1 converges to the SA forecast.

Table 7: Predicting Chicago Fed National Activity Index (CFNAI), combination weights.

\begin{tabular}{lccccccccccccc}
\hline \hline & OLS & L1 & L2 & SvmL1 & SvmL2 & EN & PLS & RF & GBRT & NN1 & NN2 & NN3 & NN4 \\
\hline SA & .077 & .077 & .077 & .077 & .077 & .077 & .077 & .077 & .077 & .077 & .077 & .077 & .077 \\
T-SA-3 &. & .100 & .100 & .100 & .100 & .100 & .100 & .100 & .100 & .100 & .100 &. &. \\
T-SA-4 &. & .111 &. & .111 & .111 & .111 & .111 & .111 & .111 & .111 & .111 &. &. \\
T-SA-5 &. & .125 &. & .125 & .125 & .125 & .125 & .125 & .125 &. & .125 &. &. \\
T-SA-6 &. & .143 &. & .143 & .143 & .143 & .143 & .143 & .143 &. &. &. &. \\
T-SA-7 &. & .167 &. & .167 & .167 & .167 &. & .167 & .167 &. &. &. &. \\
T-SA-8 &. &. &. & .200 & .200 & .200 &. & .200 & .200 &. &. &. &. \\
T-SA-9 &. &. &. & .250 &. & .250 &. & .250 & .250 &. &. &. &. \\
T-SA-10 &. &. &. & .333 &. & .333 &. & .333 &. &. &. &. &. \\
T-SA-11 &. &. &. &. &. & .500 &. & .500 &. &. &. &. &. \\
T-MCS-20\% &. &. &. & .333 &. & .333 &. & .333 &. &. &. &. &. \\
T-MCS-40\% &. &. &. &. &. & 1 &. &. &. &. &. &. &. \\
T-MCS-60\% &. &. &. &. &. & 1 &. &. &. &. &. &. &. \\
T-MCS-80\% &. &. &. &. &. & 1 &. &. &. &. &. &. \\
IL & .027 & .082 & .074 & .094 & .085 & .099 & .078 & .096 & .089 & .075 & .077 & .067 & .057 \\
CO_NNPW & .084 & .166 &. &. &. &. & .375 & .250 &. & .125 &. &. &. \\
COP1 & .076 & .077 & .077 & .077 & .077 & .077 & .077 & .077 & .077 & .077 & .077 & .077 & .077 \\
COP2 & .076 & .113 & .051 & .108 & .093 & .078 & .123 & .107 & .043 & .065 & .066 & .054 & .023 \\
COPE & .075 & .086 & .072 & .085 & .083 & .079 & .088 & .085 & .072 & .071 & .072 & .069 & .063 \\
COPS & .068 & .261 &. & .138 &. &. & .260 & .138 &. & .135 &. &. &. \\
\hline
\end{tabular}

\section{Conclusions}

Penalized constrained optimization and shrinkage techniques have been successfully used in finance; they are at the heart of asset allocation strategies.

In this paper, we point out that these methods also provide an appealing route to form forecasts combinations. We test the performances of penalized constrained optimization methods in a simulation study and two empirical applications. Our proposed strategies outperform other standard combination schemes such as the simple average, trimmed simple averages or techniques that explicitly consider individual average losses.

Methods relying on constrained optimization with penalty (COP) limit the subjectivity in the trimming phase because they do not require any predetermined selection rule or predetermined combination strategy. They only rely on the estimated covariance matrix of prediction errors. Moreover, restricting weights to be non-negative operates forecasts selection and shrinking the solution toward a target combination scheme (e.g., equal weights) improves weights stability. 
We also propose an analytical expression for the shrinkage intensity $\delta^{*}$ when targeting equal weights. This relies on the correspondence between the COP and the linear shrinkage estimator of the covariance matrix. As a matter of fact, it offers a faster alternative to computationally expensive cross-validation procedures and helps improving predictive performances when the sample size is limited relative to the number of candidate forecasts.

Acknowledgements: Francesco Roccazzella gratefully acknowledges financial support from the Economics School of Louvain. Paolo Gambetti's research is funded by the Fonds de la Recherche Scientifique (F.S.R.-FNRS) under Grant ASP FC23545. This work was supported by the Fonds de la Recherche Scientifique - (F.S.R.-FNRS) under Grant CDR J.0037.18.

\section{References}

Aiolfi, M. and Favero, C. A. (2005), 'Model uncertainty, thick modelling and the predictability of stock returns', Journal of Forecasting 24(4), 233-254.

Aruoba, S. B., Diebold, F. X., Nalewaik, J., Schorfheide, F. and Song, D. (2012), Improving u.s. GDP measurement: A forecast combination perspective, in 'Recent Advances and Future Directions in Causality, Prediction, and Specification Analysis', Springer New York, pp. 1-25.

Atiya, A. F. (2020), 'Why does forecast combination work so well?', International Journal of Forecasting 36(1), 197-200.

Ball, R. T. and Ghysels, E. (2018), 'Automated earnings forecasts: Beat analysts or combine and conquer?', Management Science 64(10), 4936-4952.

Bar-Hillel, M. (1980), 'The base-rate fallacy in probability judgments', Acta Psychologica 44(3), 211-233.

Bowley, A. L. (1920), Elements of Statistics, New York: Charles Scribner's Sons.

Claeskens, G., Magnus, J. R., Vasnev, A. L. and Wang, W. (2016), 'The forecast combination puzzle: A simple theoretical explanation', International Journal of Forecasting 32(3), 754-762.

Clemen, R. T. (1989), 'Combining forecasts: A review and annotated bibliography', International Journal of Forecasting 5(4), 559-583.

DeMiguel, V., Garlappi, L., Nogales, F. J. and Uppal, R. (2009), 'A generalized approach to portfolio optimization: Improving performance by constraining portfolio norms', Management Science 55(5), 798-812. 
Diebold, F. X. and Mariano, R. S. (1995), 'Comparing predictive accuracy', Journal of Business Ef Economic Statistics 13(3), 253-263.

Diebold, F. X. and Pauly, P. (1990), 'The use of prior information in forecast combination', International Journal of Forecasting 6(4), 503-508.

Diebold, F. X. and Shin, M. (2019), 'Machine learning for regularized survey forecast combination: Partially-egalitarian LASSO and its derivatives', International Journal of Forecasting 35(4), 1679-1691.

Fama, E. F. and French, K. R. (1989), 'Business conditions and expected returns on stocks and bonds', Journal of Financial Economics 25(1), 23-49.

Granger, C. W. J. and Ramanathan, R. (1984), 'Improved methods of combining forecasts', Journal of Forecasting 3(2), 197-204.

Granger, C. W. and Jeon, Y. (2004), 'Thick modeling', Economic Modelling 21(2), 323-343.

Green, R. C. and Hollifield, B. (1992), 'When will mean-variance efficient portfolios be well diversified?', The Journal of Finance 47(5), 1785-1809.

Greenwood, R. and Hanson, S. G. (2013), 'Issuer quality and corporate bond returns', Review of Financial Studies 26(6), 1483-1525.

Gu, S., Kelly, B. and Xiu, D. (forthcoming), 'Empirical asset pricing via machine learning', Review of Financial Studies .

Hansen, P. R., Lunde, A. and Nason, J. M. (2011), 'The model confidence set', Econometrica 79(2), 453-497.

Harrison, D. and Rubinfeld, D. L. (1978), 'Hedonic housing prices and the demand for clean air', Journal of Environmental Economics and Management 5(1), 81-102.

Jagannathan, R. and Ma, T. (2003), 'Risk reduction in large portfolios: Why imposing the wrong constraints helps', The Journal of Finance 58(4), 1651-1683.

Jurado, K., Ludvigson, S. C. and Ng, S. (2015), 'Measuring uncertainty', American Economic Review 105(3), 1177-1216.

Ledoit, O. and Wolf, M. (2003), 'Improved estimation of the covariance matrix of stock returns with an application to portfolio selection', Journal of Empirical Finance 10(5), 603621.

Ledoit, O. and Wolf, M. (2004a), 'Honey, i shrunk the sample covariance matrix', The Journal of Portfolio Management 30(4), 110-119. 
Ledoit, O. and Wolf, M. (2004b), 'A well-conditioned estimator for large-dimensional covariance matrices', Journal of Multivariate Analysis 88(2), 365-411.

Ledoit, O. and Wolf, M. (2012), 'Nonlinear shrinkage estimation of large-dimensional covariance matrices', The Annals of Statistics 40(2), 1024-1060.

Ledoit, O. and Wolf, M. (2017), 'Nonlinear shrinkage of the covariance matrix for portfolio selection: Markowitz meets goldilocks', Review of Financial Studies 30(12), 4349-4388.

Lin, H., Wu, C. and Zhou, G. (2018), 'Forecasting corporate bond returns with a large set of predictors: An iterated combination approach', Management Science 64(9), 4218-4238.

Ludvigson, S. C., Ma, S. and Ng, S. (2019), Uncertainty and business cycles: exogenous impulse or endogenous response? Working Paper.

Massey, C. and Wu, G. (2005), 'Detecting regime shifts: The causes of under- and overreaction', Management Science 51(6), 932-947.

McCracken, M. W. and Ng, S. (2016), 'FRED-MD: A monthly database for macroeconomic research', Journal of Business \&5 Economic Statistics 34(4), 574-589.

Samuels, J. D. and Sekkel, R. M. (2017), 'Model confidence sets and forecast combination', International Journal of Forecasting 33(1), 48-60.

Smith, J. and Wallis, K. F. (2009), 'A simple explanation of the forecast combination puzzle', Oxford Bulletin of Economics and Statistics 71(3), 331-355.

Timmermann, A. (2006), Chapter 4 forecast combinations, in 'Handbook of Economic Forecasting', Elsevier, pp. 135-196.

Varoquaux, G. (2018), 'Cross-validation failure: Small sample sizes lead to large error bars', NeuroImage 180, 68-77.

Wei, X. and Yang, Y. (2012), 'Robust forecast combinations', Journal of Econometrics 166(2), 224-236.

Winkler, R. L. and Makridakis, S. (1983), 'The combination of forecasts', Journal of the Royal Statistical Society. Series A (General) 146(2), 150.

Zhao, P. and Yu, B. (2006), 'On model selection consistency of lasso', Journal of Machine Learning Research 7, 2541-2563. 


\section{Appendix A. Proof of Proposition 2}

Specializing the optimization problem to the Euclidean distance between $\mathbf{w}$ and the equally weighted scheme yields (1),

$$
\boldsymbol{w}_{\delta}^{*}:=\underset{\boldsymbol{w} \in \mathcal{W}^{+}}{\operatorname{argmin}} \boldsymbol{w}^{T} \boldsymbol{\Sigma} \boldsymbol{w}+\delta\|\mathbf{w}-\mathbf{1} / m\|_{2}^{2}
$$

Developing the penalty term, making use of the fact that $\boldsymbol{w} \in \mathcal{W}^{+} \subset \mathcal{W}$ :

$$
\|\mathbf{w}-\mathbf{1} / m\|_{2}^{2}=\mathbf{w}^{T} \mathbf{w}+3 / m
$$

Discarding the constant and grouping the quadratic terms, one gets

$$
\boldsymbol{w}_{\delta}^{*}:=\underset{\boldsymbol{w} \in \mathcal{W}^{+}}{\operatorname{argmin}} \boldsymbol{w}^{T}(\boldsymbol{\Sigma}+\delta \mathbf{I}) \boldsymbol{w}
$$

which takes a similar form as (2). The proof is concluded by applying Proposition 1, replacing $\boldsymbol{\Sigma}$ by $\boldsymbol{\Sigma}+\delta \mathbf{I}$ (observe that $\boldsymbol{\Sigma}+\delta \mathbf{I}$ is positive definite whenever so is $\boldsymbol{\Sigma}$ and $\delta \geq 0$, which is always assumed to be true).

\section{Appendix B. Proof of Equivalence: general case}

The general form of the reference covariance matrix associated with the equally weighted scheme can be written as $\overline{\boldsymbol{\Sigma}}=\bar{\sigma}^{2}((1-\rho) \mathbf{I}+\rho \mathbf{E})$ where $\mathbf{E}$ is a conformable matrix of ones. It is easy to see that the optimization problem (6) associated with the specific reference matrix above collapses to that associated with $\rho=0$ under the $\mathbf{w} \in \mathcal{W}^{+}$constraint. Indeed, for every $\lambda \in[0,1[$, the objective function can be decomposed as

$$
\boldsymbol{w}^{T} \boldsymbol{\Sigma}_{\lambda} \boldsymbol{w}=\boldsymbol{w}^{T}\left(\boldsymbol{\Sigma}+\frac{\lambda}{1-\lambda}(1-\rho) \bar{\sigma}^{2} \mathbf{I}\right) \boldsymbol{w}+\frac{\lambda}{1-\lambda} \rho \bar{\sigma}^{2} \boldsymbol{w}^{T} \mathbf{E} \boldsymbol{w}
$$

On the restriction $\mathbf{w} \in \mathcal{W}^{+}$, the last term collapses to the constant $\lambda \rho \bar{\sigma}^{2} /(1-\lambda)$, which can be discarded. Hence, the cost function is equivalent to that of a COS scheme with reference matrix $\bar{\sigma}^{2} \mathbf{I}$ and shrinkage intensity $\frac{\lambda}{1-\lambda}(1-\rho)$. This shows that we can restrict ourselves to set $\rho=0$, without loss of generality. 


\section{Appendix C. Examples of the correspondence between $c$ and $\Sigma_{v}$}

Table C1: Examples of correspondences between $\boldsymbol{c}$ and $\boldsymbol{\Sigma}_{v}$ given $\alpha$.

\begin{tabular}{lcccc}
\hline \hline$\rho=0$ & Design 1 & Design 2 & Design 3 & Design 4 \\
$\alpha_{i}^{2}$ Included & 0.155 & 0.410 & 0.556 & 1 \\
$\alpha_{i}^{2}$ Excluded & 1.044 & 1.066 & 1.444 &. \\
\hline$\rho=0.25$ & & & & \\
$\alpha_{i}^{2}$ Included & 0.338 & 0.597 & 0.727 & 1 \\
$\alpha_{i}^{2}$ Excluded & 1.035 & 1.045 & 1.273 &. \\
\hline$\rho=0.5$ & & & & \\
$\alpha_{i}^{2}$ Included & 0.338 & 0.597 & 0.727 & 1 \\
$\alpha_{i}^{2}$ Excluded & 1.035 & 1.045 & 1.273 & $\cdot$ \\
\hline$\rho=0.9$ & & & & \\
$\alpha_{i}^{2}$ Included & 0.338 & 0.597 & 0.727 & 1 \\
$\alpha_{i}^{2}$ Excluded & 1.035 & 1.045 & 1.273 &. \\
\hline \hline
\end{tabular}

Notes. In the no-correlation environment $(\rho=0)$, to insure that the correspondence between $\boldsymbol{c}$ and $\boldsymbol{\Sigma}_{v}$ holds, we require the difference between included and discarded candidate forecasts to be particularly large. In particular, design 1 requires the prediction error variance of the only selected model to be 7 times smaller than the one of the excluded candidates. This is not a realistic scenario, which further questions the relevance of predictive strategies that uniquely rely on forecast selection. 


\section{Appendix D. Performance of all the considered models in the empirical studies}

Table D1: Forecasting Boston housing prices, out of sample performance.

\begin{tabular}{|c|c|c|c|c|c|c|c|c|}
\hline & \multicolumn{4}{|c|}{ Diebold-Mariano } & \multirow{2}{*}{$\begin{array}{c}\text { MCS } \\
i \in \mathcal{M}^{*}\end{array}$} & \multirow[t]{2}{*}{ RMSFE } & \multirow{2}{*}{$\begin{array}{l}\text { Time } \\
(\mathrm{sec})\end{array}$} & \multirow[t]{2}{*}{ CI } \\
\hline & COP1 & $\mathrm{COP} 2$ & COPE & COPS & & & & \\
\hline OLS & $\lll$ & $\lll$ & $\lll$ & $\lll$ & & .6273 & & \\
\hline L1 & $\lll$ & $\lll$ & $\lll$ & $\lll$ & & .6277 & & \\
\hline L2 & $\lll$ & $\lll$ & $\lll$ & $\lll$ & & .6369 & & \\
\hline svmLASSO & $\lll$ & $\lll$ & $\lll$ & $\lll$ & & .6630 & & \\
\hline svmRIDGE & $\lll$ & $\lll$ & $\lll$ & $\lll$ & & .6682 & & \\
\hline $\mathrm{EN}$ & $\ll$ & $\ll$ & $\ll$ & $\ll$ & & .6565 & & \\
\hline PLS & $\lll$ & $\lll$ & $\lll$ & $\lll$ & & .6494 & & \\
\hline $\mathrm{RF}$ & $\lll$ & $\lll$ & $\lll$ & $\lll$ & & .7136 & & \\
\hline GBRT & $\lll$ & $\lll$ & $\lll$ & $\lll$ & & .3897 & & \\
\hline NN1 & $=$ & $=$ & $\lll$ & $\ll$ & & .3519 & & \\
\hline NN2 & $=$ & $=$ & $\ll$ & $\ll$ & & .3549 & & \\
\hline NN3 & $=$ & $=$ & $\ll$ & $\ll$ & & .3558 & & \\
\hline NN4 & $=$ & $=$ & $=$ & $=$ & $*$ & .3312 & & \\
\hline $\mathrm{SA}$ & $\lll$ & $\lll$ & $\lll$ & « & & .4611 & & \\
\hline T-SA-3 & $\lll$ & $\lll$ & $\lll$ & $\lll$ & & .4314 & & $15.8 \%$ \\
\hline T-SA-4 & $\lll$ & $\lll$ & $\lll$ & $\lll$ & & .4112 & & $19.2 \%$ \\
\hline T-SA-5 & $\lll$ & $\lll$ & $\lll$ & $\lll$ & & .3878 & & $22.8 \%$ \\
\hline T-SA-6 & $\lll$ & $\lll$ & $\lll$ & $\lll$ & & .3644 & & $26.8 \%$ \\
\hline T-SA-7 & $=$ & $\ll$ & $\lll$ & $\lll$ & & .3405 & & $31.2 \%$ \\
\hline T-SA-8 & $=$ & $=$ & $=$ & $=$ & $* * *$ & .3259 & & $36.5 \%$ \\
\hline T-SA-9 & $=$ & $=$ & $=$ & $=$ & & .3369 & & $43.3 \%$ \\
\hline T-SA-10 & $=$ & $=$ & $=$ & $=$ & $*$ & .3357 & & $52.7 \%$ \\
\hline T-SA-11 & $=$ & $=$ & $=$ & $=$ & $* * *$ & .3285 & & $67.7 \%$ \\
\hline T-SA-12 & $=$ & $=$ & $=$ & $=$ & $*$ & .3312 & & $100 \%$ \\
\hline T-MCS-20\% & $=$ & $=$ & $=$ & $\begin{array}{l}-- \\
=\end{array}$ & * & .3357 & & $\overline{52 . \overline{7} \%}$ \\
\hline T-MCS- $40 \%$ & $=$ & $=$ & $=$ & $=$ & * & .3285 & & $67.7 \%$ \\
\hline T-MCS- $60 \%$ & $=$ & $=$ & $=$ & $=$ & $* * *$ & .3312 & & $100 \%$ \\
\hline T-MCS- $80 \%$ & $=$ & $=$ & $=$ & $=$ & $*$ & .3312 & & $100 \%$ \\
\hline$\overline{\mathrm{IL}}$ & $\lll$ & $\lll$ & $\lll$ & $\lll$ & & .4292 & & $5.7 \%$ \\
\hline CO_NNPW & $=$ & $\lll$ & $\lll$ & « & * & .3410 & .0801 & $\overline{41.9 \%}$ \\
\hline COP1 & & $\lll$ & $\lll$ & $\lll$ & $*$ & .3392 & 53.1800 & $32.1 \%$ \\
\hline COP2 & & & $\lll$ & $=$ & $*$ & .3307 & 31.3582 & $32.7 \%$ \\
\hline COPE & & & & $=$ & $* * *$ & .3223 & 52.8043 & $40.5 \%$ \\
\hline $\mathrm{COS}$ & & & & $=$ & $* * *$ & .3253 & .0499 & $42.6 \%$ \\
\hline
\end{tabular}

Notes. We refer to Table 4 for the details on the notation. 
Table D2: Predicting Chicago Fed National Activity Index (CFNAI), out of sample performance.

\begin{tabular}{|c|c|c|c|c|c|c|c|c|}
\hline & \multicolumn{4}{|c|}{ Diebold-Mariano } & \multirow{2}{*}{$\begin{array}{c}\text { MCS } \\
i \in \mathcal{M}^{*}\end{array}$} & \multirow[t]{2}{*}{ RMSFE } & \multirow{2}{*}{$\begin{array}{l}\text { Time } \\
(\mathrm{sec})\end{array}$} & \multirow[t]{2}{*}{ CI } \\
\hline & $\mathrm{j}: \mathrm{COP} 1$ & COP2 & COPE & COPS & & & & \\
\hline OLS & $\lll$ & $\lll$ & $\lll$ & $\lll$ & & 1.3579 & & \\
\hline L1 & $=$ & $\ll$ & $<$ & $\lll$ & & .4269 & & \\
\hline $\mathrm{L} 2$ & $=$ & $=$ & $=$ & $=$ & $* * *$ & .3534 & & \\
\hline svmLASSO & $\ll$ & $\lll$ & $\lll$ & $\lll$ & & .4065 & & \\
\hline svmRIDGE & $\ll$ & $\lll$ & $\ll$ & $\lll$ & & .4532 & & \\
\hline EN & $\lll$ & $\lll$ & $\lll$ & $\lll$ & & .4291 & & \\
\hline PLS & $\ll$ & $\lll$ & $\ll$ & $\lll$ & & .4429 & & \\
\hline $\mathrm{RF}$ & $=$ & $\lll$ & $<$ & $\ll$ & & .3983 & & \\
\hline GBRT & $\lll$ & $\lll$ & $\lll$ & $\lll$ & & .5307 & & \\
\hline NN1 & $\lll$ & $\lll$ & $\lll$ & $\lll$ & & .6794 & & \\
\hline $\mathrm{NN} 2$ & $\lll$ & $\lll$ & $\lll$ & $\lll$ & & .6658 & & \\
\hline NN3 & $\lll$ & $\lll$ & $\lll$ & $\lll$ & & .6821 & & \\
\hline NN4 & $\lll$ & $\lll$ & $\lll$ & $\lll$ & & .8212 & & \\
\hline SA & $=$ & $\lll$ & $\lll$ & $\ll$ & & .3680 & & \\
\hline T-SA-3 & $=$ & $=$ & $=$ & $=$ & $* \bar{*}$ & .3472 & & $15.8 \%$ \\
\hline T-SA-4 & $=$ & $\lll$ & $=$ & $\ll$ & & .3736 & & $19.2 \%$ \\
\hline T-SA-5 & $=$ & $<$ & $=$ & $<$ & $*$ & .3617 & & $22.8 \%$ \\
\hline T-SA-6 & $=$ & $=$ & $=$ & $<$ & & .3603 & & $26.8 \%$ \\
\hline T-SA-7 & $=$ & $<$ & $=$ & $<$ & & .3652 & & $31.2 \%$ \\
\hline T-SA-8 & $=$ & $\ll$ & $=$ & $\ll$ & & .3836 & & $36.5 \%$ \\
\hline T-SA-9 & $\lll$ & $\lll$ & $\lll$ & $\lll$ & & .4235 & & $43.3 \%$ \\
\hline T-SA-10 & $\ll$ & $\lll$ & $\lll$ & $\lll$ & & .4040 & & $52.7 \%$ \\
\hline T-SA-11 & $\ll$ & $\lll$ & $\lll$ & $\lll$ & & .4070 & & $67.7 \%$ \\
\hline T-MCS- $20 \%$ & $\ll$ & $\lll$ & $\lll$ & $\lll$ & & .4040 & & $52.7 \%$ \\
\hline T-MCS- $40 \%$ & $\lll$ & $\lll$ & $\lll$ & $\lll$ & & .4291 & & $100 \%$ \\
\hline T-MCS-60\% & $\lll$ & $\lll$ & $\lll$ & $\lll$ & & .4291 & & $100 \%$ \\
\hline T-MCS- $80 \%$ & $\lll$ & $\lll$ & $\lll$ & $\lll$ & & .4291 & & $100 \%$ \\
\hline IL & $=$ & $\ll$ & $=$ & $<$ & & .3644 & & $6.9 \%$ \\
\hline CO-NNPW & $\gg$ & $=$ & $=$ & $=$ & $\bar{*} * *$ & $.329 \overline{5}$ & 12.8071 & $43.72 \%$ \\
\hline COP1 & & $\lll$ & $\lll$ & $\ll$ & & .3680 & 110.2757 & $0.1 \%$ \\
\hline $\mathrm{COP} 2$ & & & $\ggg$ & $=$ & $* * *$ & .3360 & 144.5386 & $11.0 \%$ \\
\hline COPE & & & & $<$ & & .3566 & 207.4108 & $2.84 \%$ \\
\hline COS & & & & & $* * *$ & .3296 & .0323 & $36.0 \%$ \\
\hline COPS & & & & & $* * *$ & .3296 & .0352 & $36.0 \%$ \\
\hline
\end{tabular}

Notes. We refer to Table 4 for the details on the notation. 